\title{
Vectorial Association of Linearly Oriented Residua: Application to Swarm High-latitude Magnetic Field Measurements
}

\section{Leonie J. L. Pick ( $\square$ leonie.pick@dlr.de )}

German Aerospace Center (DLR), Institute for Solar-Terrestrial Physics https://orcid.org/0000-00025266-9764

\section{Joachim Vogt}

Jacobs University, Department of Physics and Earth Sciences

\section{Adrian Blagau}

Institute for Space Science, Bucharest

\section{Nele Stachlys}

University of Potsdam, Institute of Physics and Astronomy

\section{Research Article}

Keywords: Correlation analysis, field-aligned currents, current sheet polarization, Swarm, auroral oval, magnetosphereionosphere coupling, magnetic field perturbation

Posted Date: May 5th, 2021

DOI: https://doi.org/10.21203/rs.3.rs-461442/v1

License: (c) (i) This work is licensed under a Creative Commons Attribution 4.0 International License. Read Full License 


\section{Vectorial Association of Linearly Oriented Residua (VALOR):}

\section{Application to Swarm high-latitude magnetic field measurements}

3 Leonie Pick (corresponding author), Department of Space Weather Impact, Institute for Solar-Terrestrial Physics, German Aerospace Center, 53 Kalkhorstweg, Neustrelitz, 17235, Germany, leonie.pick@dlr.de

Joachim Vogt, Department of Physics and Earth Sciences, Jacobs University, 1 Campus Ring, Bremen, 28759, Germany, j.vogt@jacobs-university.de

Adrian Blăgău, Institute for Space Science, Bucharest, 409 Atomistilor Street, Magurele, Ilfov, 077125, Romania, blagau@spacescience.ro

Nele Stachlys, Faculty of Science, Institute of Physics and Astronomy, University of Potsdam, stachlys@uni-potsdam.de 


\section{Abstract}

6 We introduce the methodological framework VALOR for the analysis of field-aligned current (FAC)

7 sheets based on dual-spacecraft magnetic field observations. VALOR generalizes existing approaches in

8 two respects. First, it takes advantage of the primary observables at both spacecraft, namely, the full

9 magnetic field vectors, instead of derived scalar quantities like single- or dual-spacecraft FAC densities.

10 Second, rather than being restricted to linear correlation measures, VALOR allows to define a

11 custom-made statistical measure of association between the signals at both spacecraft, that is chosen to

12 be particularly sensitive to small-scale fluctuations for this first demonstration. Additionally, VALOR

13 offers to consider the linear orientation of the magnetic field residual vectors, i.e., the sheets'

14 polarization, in order to infer their orientation and to accentuate the association measure. This paper

15 illustrates the method on the basis of an exemplary auroral oval crossing on March 1, 2019 by the lower

16 pair of ESA's multi-spacecraft mission Swarm. Explicit comparisons to competing methods are

17 performed to give credence to the results of the case study while the general capability of VALOR is

18 demonstrated on the basis of a statistical study comprising around 9700 auroral oval crossings from

19 2014-2020. One suitable area of application is the analysis of meso- to small-scale (tens of km to below

20 one $\mathrm{km}$ ) auroral arc systems in combination with optical data from modern all-sky imagers.

${ }_{21}$ Conceptually, VALOR is neither bound to the auroral zone nor to Swarm and can be applied by any

22 multi-spacecraft geospace mission with suitable instrumentation and orbit configuration.

\section{Keywords}

24 Correlation analysis, field-aligned currents, current sheet polarization, Swarm, auroral oval, magnetosphere25 ionosphere coupling, magnetic field perturbation

\section{Introduction}

27 Electromagnetic magnetosphere-ionosphere (M-I) coupling processes are optically manifested through ${ }_{28}$ auroral phenomena in the high-latitude ionosphere, with their occurrence frequency maximizing in oval29 shaped region around the geomagnetic poles. Auroral emissions exhibit a variety of colors, shapes, 30 and dynamics. Among the best studied forms are elongated linear structures called auroral arcs where 
44 scales $L \gtrsim 10 \mathrm{~km}$ (Vogt and Haerendel 1998$)$.

45 Auroral FACs are routinely measured using magnetic field instruments on Earth-orbiting satellites such

46 as Magsat, Freia, FAST, Cluster, Champ, ST5, and Swarm. For recent reviews of studies on auroral FACs

47 and their spatial scale distributions, see the introductory sections of Blăgău and Vogt (2019) and Bunescu

48 et al (2019). Apart from the dual-spacecraft gradient technique applied to the low-pass filtered and thus

49 large-scale magnetic field data of the lower pair of Swarm satellites (Ritter and Lühr 2006) Ritter et al

thickness indicates the spatial scale of the underlying physical process. Statistical studies reveal a wide spectrum of arc scales ranging from about hundred meters (Maggs and Davis 1968) to several hundreds of kilometers, comparable to the width of the auroral oval. While narrow or small-scale auroral arcs with scales in the range of hundred meters to a kilometer are typically very dynamic and short-lived (Sandahl et al 2008), auroral arcs in the mesoscale range of a few ten kilometers are both rather frequent (Knudsen et al (2010), optical instrument characteristics can strongly influence the measured scale distribution.

Auroral emissions are closely associated with electric currents flowing along geomagnetic field lines (e.g., Lysak 1990 Paschmann et al 2002 Vogt 2002). Such field-aligned currents (FACs) transmit energy and momentum between remote regions in the near-Earth space environment, and thus serve as important coupling agents facilitating global-scale plasma convection and magnetospheric substorms. The spatial scale $L$ of FACs serves as an indicator for the type of M-I coupling, with quasi-static coupling associated with large scales $L \gtrsim 100 \mathrm{~km}($ Lyons 1980 , Lotko et al 1987), and Alfvénic coupling with intermediate 2013, Vogt et al 2013, 2020), FAC scale analyses rest on finite magnetic field differences along the tracks of single spacecraft (Lühr et al 1996, Ritter and Lühr 2006). The scalar FAC density estimate can then be differentiated with regard to spatial scales by means of appropriate filters (Forsyth et al 2017) or transformation techniques (Stasiewicz and Potemra 1998). The multiscale FAC analyzer introduced by Bunescu et al $2015,2017,2019)$ produces wavelet-like scalograms not only for the FAC density but also for the planarity and the orientation of FAC structures.

In the case of multi-spacecraft missions such as ST5 or Swarm, scale-dependent patterns in FAC density profiles measured at different locations can be statistically compared to infer their degree of association. The most popular association measure is linear correlation, employed in the auroral FAC context to study the scale dependence of day-night differences (Gjerloev et al 2011), the temporal variability of FAC structures (Lühr et al 2015), the validity of the single-spacecraft FAC estimator (Forsyth et al 
61 87 crossings. orientation.

\section{Methods}

2017), characteristics and controling parameters (McGranaghan et al 2017), and auroral current sheet orientations (Yang et al 2018).

This paper introduces the methodological framework VALOR (Vectorial Association of Linearly Oriented Residua) to extend the toolbox of multi-spacecraft correlation analysis techniques that have so far been applied to Swarm observations of auroral FAC structures. Although VALOR builds on general multispacecraft analysis theory (Vogt et al 2011, 2013, Vogt 2014), we have in mind the specific constellation of Swarm satellites Alpha ( $\mathrm{SwA})$ and Charlie $(\mathrm{SwC})$. We are particularly interested in the study of intermediate to small spatial scales down to a few kilometers, hopefully involving also measurements at a higher resolution than one Hz. To this end, VALOR is designed to extract scale information with as little assumptions about filter shapes or scale-dependent basis functions as possible. Instead of using the derived Level-2 scalar single-spacecraft or dual-spacecraft FAC densities, VALOR is based on the full Level-1b magnetic field vector to construct maps of suitable statistical association measures. VALOR is not restricted to the class of linear correlation techniques, and can explicitly consider signal polarization at an auroral current sheet, i.e., the directional preference of the magnetic field vector, to infer the sheet

We proceed as follows. In the Methods section, the VALOR approach is developed and illustrated on the basis of an exemplary auroral oval crossing by the lower pair of Swarm spacecraft. The section closes with an explanation of how the data base for the multi-event study is compiled. The Results section presents a detailed evaluation of the case study followed by the statistical results. The same structure is kept throughout the Discussion $\mathscr{G}$ Outlook section where we explicitly compare VALOR to related methods and give an outlook on future work before we present our Conclusions.

To provide context and a reference analysis technique, linear cross-correlation analysis is summarized before the VALOR methodology is explained. The general VALOR framework for vectorial time series is presented, and then we discuss how polarization information comes into play. The rest of the section is concerned with data selection and preprocessing in preparation of our statistical study of auroral crossings. 


\section{Linear correlation analysis}

Linear (cross-)correlation analysis rests on the linear correlation coefficient $r=r\left(X, Y_{\tau}\right)$ between one signal $X=X(t)$ and a lagged version of another signal $Y_{\tau}=Y(t+\tau)$. In the context of Swarm auroral FAC density studies, Forsyth et al (2017) pointed out that $r$ quantifies only the degree of linear dependence between the two signals. Additionally, they considered the slope $m=m\left(X, Y_{\tau}\right)$ of the regression line to study if the amplitudes of the signals are comparable, and argued that both $r \approx 1$ and $m \approx 1$ are required for a good match of two FAC density profiles.

As shown in Appendix A, the two quality parameters $r$ and $m$ can be combined into a single measure of signal association, namely, the mean squared deviation (MSD) of $X$ and $Y_{\tau}$ :

$$
\operatorname{MSD}\left(X, Y_{\tau}\right)=\left\langle\left|X(t)-Y_{\tau}(t)\right|^{2}\right\rangle_{t}=(\Delta X)^{2}\left(1-r^{2}\right)+\left(\Delta Y_{\tau}\right)^{2}(1-m)^{2}+\left(\bar{X}-\bar{Y}_{\tau}\right)^{2}
$$

Here, $\langle\bullet\rangle_{t}$ and $\boldsymbol{\bullet}$ both denote time averaging, and $(\Delta X)^{2}$ and $\left(\Delta Y_{\tau}\right)^{2}$ are the variances of the two signals. Hence for the MSD to be small, both $r$ and $m$ must be close to one, and the time series means $\bar{X}$ and $\bar{Y}_{\tau}$ must not differ much. The third term cancels if centered time series $X_{c}=X-\bar{X}$ and $Y_{\tau, c}=Y_{\tau}-\bar{Y}_{\tau}$ are considered instead (see Appendix A). With the MSD being close to zero for a good match the inverse MSD as a function of $\tau$ is expected to display a clear peak at optimum lag $\tau_{0}$. This idea is picked up by the VALOR methodology with respect to the construction of an association measure as a function of both $t$ and $\tau$.

\section{The VALOR analysis framework}

A straightforward generalization of the MSD from the scalar to the vectorial case gives $\operatorname{MSD}\left(\boldsymbol{X}, \boldsymbol{Y}_{\tau}\right)=$ $\left\langle\left\|\boldsymbol{X}(t)-\boldsymbol{Y}_{\tau}(t)\right\|^{2}\right\rangle_{t}$ where $\|\bullet\|$ is the standard vector norm. For the sake of symmetry the lagged term $\boldsymbol{Y}_{\tau}(t)$ is replaced by a pattern function $\boldsymbol{P}=\boldsymbol{P}(t \mid \tau)$ constructed from both signals. VALOR can then be understood as the special dual-satellite case of the general multi-spacecraft analysis framework suggested by Vogt 2014, Chapter 5) for estimating boundary parameters from vectorial magnetic time series.

Here, $\boldsymbol{X}(t)$ and $\boldsymbol{Y}(t)$ refer to the ionospheric magnetic perturbations $\left(\boldsymbol{b}_{\sigma}\right)$ registered at SwA $(\sigma=1)$ and SwC $(\sigma=2)$ as they traverse the auroral ovals. The perturbations are extracted from the vector field magnetometer data $\left(\boldsymbol{B}_{\sigma}\right.$, Level-1b, MAG[A/C]_LR_1B) recorded at 1 Hz resolution:

$$
\boldsymbol{b}_{\sigma}(t)=\boldsymbol{B}_{\sigma}(t)-\boldsymbol{B}^{*}\left(\boldsymbol{r}_{\sigma}(t)\right) \quad \sigma=1,2 .
$$


The ambient magnetic field $\left(\boldsymbol{B}^{*}\right)$ is modeled as a superposition of contributions from the core, the lithospheric and the magnetospheric magnetic fields and evaluated at the spacecraft positions $\left(\boldsymbol{r}_{\sigma}\right)$ using predictions from CHAOS-7 (Finlay et al 2020$)$.

The magnetic perturbations during one such auroral oval crossing on March 1, 2019 around 02:00 UTC are shown in Figure 17a where they are given in the geographic North (N) - East (E) - Center (C) local Cartesian coordinate frame (NEC). The two spacecraft record very similar signatures with the SwC time series being lagged by the difference in equatorial crossing times $\left(\tau_{\mathrm{eq}}=-6 \mathrm{~s}\right)$ for the purpose of this illustration. The distinct disturbance patterns evident in the East components (orange/red) indicate that SwA and SwC cross two roughly East-West oriented current sheets on their outbound (or equatorward) orbits: first a downward (or earthward) and then an upward directed FAC in accordance with the canonical Region-1/Region-2 morphology of large-scale auroral FACs (Iijima and Potemra 1976, He et al 2012 , 2014). This is clearly seen in the bipolar FAC density profiles shown in Figure 1 1 (derivation in Ritter et al 2013). Obviously, the small-scale variability contained in the Level-1b single-spacecraft estimators $\left(j_{\sigma}, \mathrm{FAC}[\mathrm{A} / \mathrm{C}] \mathrm{TMS} \_2 \mathrm{~F}\right.$, blue) is largely subdued in the Level-2 dual-spacecraft estimator $\left(j_{12}\right.$, FAC_TMS_2F, orange) following the low-pass filtering (cutoff period at $20 \mathrm{~s}$ ) of $\boldsymbol{b}_{\sigma}$ prior to its calculation. Since the $j_{\sigma}$ estimators are more closely related to along-track differences of $\boldsymbol{b}_{\sigma}$ than to $\boldsymbol{b}_{\sigma}$ themselves, the pattern function $\boldsymbol{P}$ is constructed as an average of temporal derivatives $\dot{\boldsymbol{b}}_{\sigma}$ (obtained using centered finite differencing)

$$
\boldsymbol{P}(t \mid \tau)=\frac{1}{2}\left[\dot{\boldsymbol{b}}_{1}(t)+\dot{\boldsymbol{b}}_{2, \tau}(t)\right],
$$

which provides a parametric family of reference perturbation signatures in the centered spacecraft frame. Here, the time series are restricted to a window $t=\left[-t_{\max }, \ldots, t_{\max }=150 \mathrm{~s}\right]$ selected based on a probabilistic interpretation of the normalized $j_{12}$ magnitude (Fig. 11; details in the end of the section). Considering a range of $\mathrm{SwC}$ orbital lags with respect to $\mathrm{SwA}, \tau=\left[-\tau_{\max }, \ldots, \tau_{\max }=20 \mathrm{~s}\right]$, the pattern function is evaluated over a $2 t_{\max }-2 \tau_{\max }+1=261 \mathrm{~s} \times 41 \mathrm{~s}$ sampling domain.

The $t \mid \tau$-sampling domain is queried with respect to the optimum spacecraft lag $\left(\tau_{0}\right)$ as well as the center $\left(t_{0}\right)$ and spatial scale $(L)$ of the FAC signature. These tasks are conveniently addressed by evaluating the scalar association measure

$$
\mathcal{C}(t \mid \tau)=\frac{\|\boldsymbol{P}(t \mid \tau)\|^{2}}{\operatorname{MSD}\left(\dot{\boldsymbol{b}}_{1}, \boldsymbol{P}\right)}=\frac{\|\boldsymbol{P}(t \mid \tau)\|^{2}}{\left\langle\left\|\dot{\boldsymbol{b}}_{1}(t)-\boldsymbol{P}(t \mid \tau)\right\|^{2}\right\rangle_{t}}
$$


According to our preceding deliberation $\tau_{0}$ is the element of the $\tau$-sampling domain at which $\mathcal{C}$ maximizes:

$$
\tau_{0}=\underset{\tau}{\arg \max } C(t \mid \tau)
$$

Statistically robust estimates of $t_{0}$ and $L$, i.e., ones with limited dependency on the specific form of $\boldsymbol{P}\left(t \mid \tau_{0}\right)$, are obtained by interpreting the normalized association measure at optimum lag $\mathcal{C}\left(t \mid \tau_{0}\right)$ as probability density function (PDF) with quartiles Q1, Q2 (median) and Q3:

$$
\begin{aligned}
t_{0} & =\mathrm{Q} 2 \\
L & =\mathrm{IQR} \cdot\|\boldsymbol{v}\|=(\mathrm{Q} 3-\mathrm{Q} 1) \cdot\|\boldsymbol{v}\| \\
\boldsymbol{v} & =-\frac{1}{2}\left[\boldsymbol{v}_{1}\left(t_{0}\right)+\boldsymbol{v}_{2, \tau_{0}}\left(t_{0}\right)\right]
\end{aligned}
$$

Here, IQR is the interquartile range and $\boldsymbol{v}$ is the FAC velocity in the centered spacecraft frame calculated under the assumption that the FAC doesn't move with respect to the ground. The spacecraft velocities $\boldsymbol{v}_{\sigma}$ are obtained from centered finite differences of the respective positions $\boldsymbol{r}_{\sigma}$.

Note that the VALOR framework is not dependent on a particular choice of association measure. The one introduced here (Eqs. 3, 4) uses the magnetic perturbations at SwA $(\sigma=1)$ as a reference and is tailored to the identification of small-scale FAC characteristics.

\section{Magnetic polarization at FAC sheets}

In addition to being capable of processing vectorial time series and selecting a wider class of association measures than standard linear correlation techniques, VALOR may explicitly address the current sheet's orientation by estimating a triplet of unit vectors $\{\hat{\boldsymbol{q}}, \hat{\boldsymbol{n}}, \hat{\boldsymbol{p}}\}$. Here, $\hat{\boldsymbol{q}}=\boldsymbol{q} /\|\boldsymbol{q}\|$ is the ambient magnetic field direction in the centered spacecraft frame obtained from a magnetic field model $\boldsymbol{B}^{*}$ (see Eq. 2) as follows:

$$
\boldsymbol{q}(t \mid \tau)=\frac{1}{2}\left[\boldsymbol{B}^{*}\left(\boldsymbol{r}_{1}(t)\right)+\boldsymbol{B}^{*}\left(\boldsymbol{r}_{2, \tau}(t)\right)\right]
$$

In an ideal planar FAC sheet geometry, magnetic perturbation vectors are perpendicular to both $\hat{\boldsymbol{q}}$ and the sheet normal unit vector $\hat{\boldsymbol{n}}$, thus $\hat{\boldsymbol{p}}=\hat{\boldsymbol{q}} \times \hat{\boldsymbol{n}}$ is the direction of the associated linear polarization of the magnetic perturbation vector. Candidate unit vectors $\hat{\boldsymbol{n}}_{c}$ and $\hat{\boldsymbol{p}}_{c}$ are constructed from differences in SwA and SwC spacecraft locations $\boldsymbol{\Delta} \boldsymbol{r}_{12}$ according to (see Fig. 2)

$$
\begin{aligned}
\hat{\boldsymbol{n}}_{c}(t \mid \tau) & =\left(\frac{\boldsymbol{\Delta} \boldsymbol{r}_{12}(t \mid \tau) \times \hat{\boldsymbol{q}}(t \mid \tau)}{\left\|\boldsymbol{\Delta} \boldsymbol{r}_{12}(t \mid \tau) \times \hat{\boldsymbol{q}}(t \mid \tau)\right\|}\right) \text { with } \quad \boldsymbol{\Delta} \boldsymbol{r}_{12}(t \mid \tau)=\boldsymbol{r}_{2, \tau}(t)-\boldsymbol{r}_{1}(t), \\
\hat{\boldsymbol{p}}_{c}(t \mid \tau) & =\hat{\boldsymbol{q}}(t \mid \tau) \times \hat{\boldsymbol{n}}_{c}(t \mid \tau) .
\end{aligned}
$$


We expect the association measure (Eq. 4) to further accentuate when the current sheet polarization is taken into account in the construction of an improved pattern function $\boldsymbol{P}_{p}$. To this end, $\boldsymbol{P}=\boldsymbol{P}(t \mid \tau)$ is projected onto the candidate polarization vector $\hat{\boldsymbol{p}}_{c}$ to yield

$$
\boldsymbol{P}(t \mid \tau)_{p}=\left[\boldsymbol{P}(t \mid \tau) \cdot \hat{\boldsymbol{p}}_{c}(t \mid \tau)\right] \hat{\boldsymbol{p}}_{c}(t \mid \tau)
$$

The anticipated merit of this projection is to reduce contributions from incorrect candidate polarization directions to the association measure. Once the final triplet $\left(\hat{\boldsymbol{q}}, \hat{\boldsymbol{n}}_{0}, \hat{\boldsymbol{p}}_{0}\right)$ has been selected from the candidates based on $\left(t_{0}, \tau_{0}\right)$, the FAC's spatial scale in the direction of $\hat{\boldsymbol{n}}_{0}$ can be calculated as

$$
L_{n}=\mathrm{IQR} \cdot\left\|\left[\boldsymbol{v} \cdot \hat{\boldsymbol{n}}_{0}\left(t_{0} \mid \tau_{0}\right)\right] \hat{\boldsymbol{n}}_{0}\right\|,
$$

using the updated IQR and leaving $\boldsymbol{v}$ (Eq. 8) unchanged.

\section{Preprocessing for multi-event analysis}

Apart from the demonstration of VALOR's performance on the basis of a sample event a proper assessment of the method also requires its evaluation in a statistical sense. Theoretically, given four auroral oval crossings per orbit with an orbital period of $\sim 93.3$ minutes for Swarm, we can expect $\sim 135 \cdot 10^{3}$ crossing events within the six year long analysis time span from April 2014 to April 2020. Here, we apply VALOR to a reduced set of suitable auroral FAC signatures observable in the $1 \mathrm{~Hz}$ low resolution data as a first step.

First, we extract the polar segments from all orbits during the analysis span by restricting the spacecrafts' quasi-dipole (QD, see Laundal and Richmond 2017) latitude to $\left|\theta_{m}\right|>50^{\circ}$. Then, we require that (1) the polar orbit segments pass close to either one of the QD poles, $\max \left(\left|\theta_{m}\right|\right)>88^{\circ}$, in order to ensure quasi-perpendicular passages through the current sheets and thus small inclinations $\alpha$ (cf., Fig. 2b), (2) full records of the dual-spacecraft FAC density product $j_{12}$ are available along the selected segments in order to specify the VALOR analysis windows (details below). Finally, the remaining orbit segments are split at $\max \left(\left|\theta_{m}\right|\right)$ into inbound (poleward) and outbound (equatorward) orbital arcs. While the first requirement accounts for a reduction of $\sim 86 \%$ with respect to the theoretically determined number of events the second one halves the remaining record. This leaves $9709(\sim 7 \%)$ auroral oval crossings in the final set of which the sample event in Figure 1 is event \#7842.

We standardize the selected orbital windows prior to the application of VALOR (Eqs. 2 13) on the basis of the $j_{12}$ time series. Their normalized magnitudes constitute PDFs along the arcs with the associated 
medians (Q2) and IQRs being robust initial estimators of the FAC signatures' centers $\left(t_{0 i}\right)$ and length scales $\left(L_{i}\right)$, respectively (as in Eqs. 6] 8). Given a median IQR of $86 \mathrm{~s}\left(\tilde{L}_{i} \sim 650 \mathrm{~km}\right)$ across all 9709 events, we decide to select $300 \mathrm{~s}$ long windows centered on $t_{0 i}$ (just 23 events with IQR $>300 \mathrm{~s}$ ) which are now free to extend below $\left|\theta_{m}\right|=50^{\circ}$. Following the scope of event comparability the subsequent VALOR analysis is performed in the global Cartesian geographic coordinate frame (NEC $\rightarrow$ GEO; see Laundal and Richmond 2017, p. 29) in which the magnetic perturbations $\boldsymbol{b}_{\sigma}$ are set to zero whenever the event windows extend beyond the QD poles in order to avoid confusion of consecutive events.

Lastly, note that the selection scheme outlined here allows not only for typical single-peak magnetic perturbation profiles (as evident in event \#7842) but also for different noisy profiles including sigmoidal (single FAC crossing) and multi-peak signatures ( $>$ two crossings). This needs to be taken into account when interpreting VALOR's results.

\section{Results}

In this section we first present the VALOR results for the sample event \#7842 (2019/03/01 01:58:4902:03:49 UT) before we put them into perspective in the context of the statistical results obtained on the basis of all 9707 selected FAC crossings.

\section{The sample event}

According to the preprocessing the analysis window is centered on $t_{0 i}=02: 01: 19 \mathrm{UT}$, when SwA is located at $\theta_{m}=66.3^{\circ} \mathrm{QD}$ latitude and MLT $=21.655$ magnetic local time (see Laundal and Richmond 2017, p. 51), and the initial FAC scale length is $L_{i} \sim 626 \mathrm{~km}$ (IQR $=82 \mathrm{~s}$, see Fig. 1 1 ).

These estimates are updated in the VALOR framework on the basis of the association measure (Eq. (4) as illustrated in Figure 3. The plot compares three association measures based on different kinds of input: Scalar $j_{\sigma}$ instead of $\dot{\boldsymbol{b}}_{\sigma}\left(\mathcal{C}_{s}\right.$, panels a \& d), vector $\dot{\boldsymbol{b}}_{\sigma}\left(\mathcal{C}_{v}\right.$, panels b \& e), and the final combination of vector $\dot{\boldsymbol{b}}_{\sigma}$ and $\hat{\boldsymbol{p}}_{c}\left(\mathcal{C}_{p}\right.$, panels c \& f). We acknowledge that the consideration of polarization information helps to constrain the association measure with respect to $\tau$ as expected (Fig. 3p vs. 35). At the same time we notice that $\mathcal{C}(t \mid \tau)$ maximize at $\tau_{0}$ that diverge notably between the "scalar" case on the one hand and the "vector only" and "polarization" cases on the other (dashed horizontal lines, Fig. 33 vs. $3 \mathrm{~b}$ \& 32). For the latter the corresponding $\mathcal{C}\left(t \mid \tau_{0}\right)$-based PDFs are better focused around the medians $\left(t_{0}\right.$, dashed vertical lines) yielding smaller IQRs and thereby spatial scales (Fig. 3 $3 \mathrm{~d}$ vs. 3e \& $3 \mathrm{f}$ ). Using both 
polarization and vector information in the final setup VALOR gives a $\mathcal{C}\left(t \mid \tau_{0}=-11 \mathrm{~s}\right)_{p}$ derived median 15 $\mathrm{s}$ after $t_{0 i}\left(t_{0}=02: 01: 34 \mathrm{UT}\right)$, corresponding to $\theta_{m}=65.48^{\circ}$ and MLT $=21.72$ in centered spacecraft coordinates, as well as a scale length $L_{n} \sim 345 \mathrm{~km}(\mathrm{IQR}=49 \mathrm{~s}$, Fig. 3i $)$ roughly half the size of $L_{i}$. If not stated otherwise we will refer to this setup in the following.

Regarding the geometry the sample event presents a favorable case as the spacecraft tangential plane roughly coincides with the $\hat{\boldsymbol{q}}_{\perp}$-plane (dihedral angle $\beta=\measuredangle\{\hat{\boldsymbol{r}}, \hat{\boldsymbol{q}}\}=167^{\circ}$, see Fig. 22b). The latter is approximated by the X-Y-plane of the mean field-aligned coordinate system (MFA) in Figure 4a, where the Z-axis is aligned with the mean ambient magnetic field $\left(\bar{B}^{*}\right)$ at the centered spacecraft locations from start (s) to end (e), and the Y-axis points towards local magnetic East. Lines of MLT (20, 22) and QD latitude $\left(60^{\circ}, 70^{\circ}\right)$ provide spatial orientation in accordance with Figure 1 .

Apparently, the pattern function $\boldsymbol{P}\left(t \mid \tau_{0}=-11 \mathrm{~s}\right)_{p}$ (colored arrows) is polarized roughly along local magnetic West $\left(\gamma=\measuredangle\left\{[0,1,0], \hat{\boldsymbol{p}}_{0_{\mathrm{MFA}}}\right\}=163^{\circ}\right)$ which is consistent with a relatively small inclination $(\alpha=$ $\left.\measuredangle\left\{\hat{\boldsymbol{v}}_{\hat{\boldsymbol{q}}_{\perp}}, \hat{\boldsymbol{n}}_{0}\right\}=19^{\circ}\right)$ as imposed via the event selection scheme. The sign reversal evident shortly after reaching $t_{0}$ (red dot) is indicative of the transition from the downward to the upward oriented current sheet (c.f., Fig. 11 1 ,b). Note that the agreement between $t_{0}$ and the position of maximum pattern amplitude $(27 \mathrm{nT} / \mathrm{s})$ is not generally implied by the VALOR technique but rather a peculiarity of the sample event arising from the asymmetry of the underlying magnetic perturbation signatures. Given the common bi-modal current structure, $t_{0}$ may be interpreted as a proxy for the auroral oval center. Accordingly, $L_{n}$ refers to the inter-FAC distance as a typical length scale of the auroral oval.

Furthermore, event \#7842 presents a suitable sample case because SwA and SwC pass almost centrally through the field of view (FOV; gray ellipse in Fig. 4a ) of the THEMIS All-Sky-Imager (ASI) at Kuujjuaq, Canada ( $58.1^{\circ}$ geographic latitude; $291.5^{\circ}$ longitude). The ASI camera takes $256 \times 256$ pixel resolved white light images of the night sky at $3 \mathrm{~s}$ cadence (Mende et al 2008). In order to summarize the recorded auroral activity one pixel wide North-South slices at the FOV's center are stacked in time with $6 \mathrm{~s}$ cadence forming a keogram (Fig. 4b). During event \#7842 the keogram shows a distinct auroral structure (marked by blue lines) which moves southward at roughly $0.64 \mathrm{~km} / \mathrm{s}$. This is smaller than $10 \%$ of the centered spacecraft velocity $(\|\boldsymbol{v}\|=7.63 \mathrm{~km} / \mathrm{s})$ so that the assumption on the FAC's stationarity is justified in this case. 


\section{The set of selected events $04 / 2014-03 / 2020$}

The VALOR results for the 9709 preselected FAC crossings are summarized in form of nine histograms in Figure 5. The sample event (dashed lines) is representative of FACs registered around midnight that are typically centered at lower latitudes $\left(\theta_{m} \sim 69^{\circ}\right)$ than those registered around noon $\left(\theta_{m} \sim 78^{\circ} ;\right.$ Fig. 5 p). The larger event counts at noon and midnight together with the relatively equal sampling of MLTs (Fig. 5b) indicate that the distribution is more narrowly confined in latitude compared to dawn and dusk where current sheets are spread between $\theta_{m} \sim 62^{\circ}$ and $85^{\circ}$. Note that these positions are based on VALOR-derived optimum spacecraft lags which follow a normal distribution around $\overline{\tau_{0}}=-8 \mathrm{~s}$ (Fig. 5 , gray) that notably differs from the distribution formed by the offsets in equatorial crossing times $\left(\tau_{\text {eq }}\right.$, black).

Regarding the geometrical considerations the statistical results show that the favorable condition of a relatively small dihedral angle holds in general $\left(\beta \leq 17^{\circ}\right.$, Fig. $\left.5 \mathrm{~d}\right)$. This lends credence to the estimated orientations according to which the FAC sheets are closely oriented along the magnetic EastWest direction (median $\tilde{\gamma}=17^{\circ}$, Fig. 5p) and are thus crossed quasi-perpendicularly $\left(\tilde{\alpha}=17^{\circ}\right.$, Fig. 5 f $)$ as expected. The angle intervals $\left[0^{\circ}-90\right)^{\circ}$ and $\left[90^{\circ}, 180^{\circ}\right)$ are overlain as stacked histograms (cyan, magenta in Fig. 5d-f) and reveal asymmetries caused by the hemispheric differences in magnetic field morphology and a relative oversampling of northern-hemispheric events (70\%). However, the distribution of inclinations is fairly symmetric (Fig. 5f) owing to the even sampling of events on inbound and outbound orbits. For instance, there are about as many East-West polarized, northern hemispheric sheets encountered on outbound orbits (" $\hat{\boldsymbol{p}}$ twds W, out N" like sample event) as on inbound ones.

The consideration of the polarization (Eq. 12 in the association measure results in a general reduction of the FAC spatial scales by $\sim 40 \mathrm{~km}$ to $\tilde{L}_{n}=227 \mathrm{~km}$ compared to the "scalar" $\left(L_{s}\right)$ and "vector only" $\left(L_{v}\right)$ cases (Fig. 5g, gray vs. blue \& red). As expected, this is significantly less than $\tilde{L}_{i}=656 \mathrm{~km}$ obtained from the $j_{12}$ based preprocessing (black). The resolution power of the latter is limited to $L_{i} \geq 150 \mathrm{~km}$ because, in contrast to VALOR, the underlying magnetic field residuals are low-pass filtered (cf., Fig. 11p). The obtained statistics depend on the chosen association measure (Fig. 5h, Eq. 44 in that different FAC spatial scales provoke systematically different responses. This becomes evident when the histograms are split into small $\left(L_{n}<150 \mathrm{~km}\right)$, medium $\left(150 \leq L_{n} \leq 300 \mathrm{~km}\right)$ and large $\left(L_{n} \geq 300 \mathrm{~km}\right)$ scale subclasses (Fig. 6, not stacked): Large scales are most prevalent at dawn/dusk MLT sectors with relatively small similarities $\left(\tilde{\mathcal{C}}_{p}=7\right)$ while small scales dominates at noon $/$ midnight with larger similarities $\left(\tilde{\mathcal{C}}_{p}=41\right)$ and 
the medium scales fall in-between (Fig. 6b, g, h). In that sense the sample event is a typical medium to large scale hybrid.

However, the amplitude of the association measure at the selected center points does not readily lend itself to a quantitative assessment of the results' validity, i.e., large-scale structures are not per se less eligible for the VALOR analysis. In fact, one possibility to assess the fundamental assumption of sheet-like current geometries is via the "planarity" measure $N$, i.e., the mean ratio of variances of $\boldsymbol{b}$ projected onto the $\hat{\boldsymbol{p}}_{0}$ and $\hat{\boldsymbol{n}}_{0}$ directions (Fig. 5). Truly planar structures should give planarity measures significantly larger than one which holds for the majority of cases $(\tilde{N}=18.6 ; 20 \% \leq 5)$. Here, it is the large-scale fraction that scores best although by a smaller margin (Fig. 6. $)$.

\section{Discussion and Outlook}

Following the logic of the previous section our discussion is structured into two parts: First, we directly compare VALOR to linear correlation and regression analysis as well as Minimum Variance Analysis by means of the sample event. We then highlight to what extent our statistical results reflect the known characteristics of multiscale auroral FACs and comment on the influence of our event selection scheme.

Finally, we give thought to the future development and application of the VALOR method.

In the beginning we have demonstrated how VALOR emerges from the combination and generalization of scalar association measures defined in both linear cross-correlation $(r)$ and regression analysis $(m)$. Following Forsyth et al (2017) we have evaluated both parameters for the sample event in order to substantiate the capability of VALOR (Fig. 7). The reference analysis indicates pronounced similarity $(r>0.9 \vee 0.9<m<1.1)$ of the $40-50 \mathrm{~s}$ bandpass filtered single-spacecraft current densities $j_{\sigma}$ for two distinct time periods (around 02:00:00 and 02:02:30 UT) consistent with the consecutive crossing of two large-scale $(\sim 300-380 \mathrm{~km})$ FAC sheets (cf., Fig. 1p). On the other hand VALOR detects relatively high levels of association in the FAC transition zone $\left(t_{0}=02: 01: 34 \mathrm{UT}\right.$, auroral oval center $)$ which persist on a comparable spatial scale $\left(L_{n}=345 \mathrm{~km}\right)$. The difference in the positioning results from VALOR's sensitivity to aligned small-scale amplitude fluctuations in $\dot{\boldsymbol{b}}_{\sigma}$ (large $\left\|\boldsymbol{P}\left(t, \tau_{0}\right)\right\|$ ) and the fact that one position is calculated in the presence of two current sheets. Actually, if one applies VALOR separately to the periods before and after $t_{0}$ the resulting sheet centers (and widths) are consistent with the reference analysis (Fig. 8). This experiment also shows that different optimum spacecraft lags $(-4 \mathrm{~s}$ and $-10 \mathrm{~s})$ can be estimated for adjacent FAC crossings $\left(\tau_{0}=-11 \mathrm{~s}\right)$. The ability to adapt to different crossing 
geometries (cf., Fig. 2) is a profound advantage of VALOR compared to the reference technique which, given that it operates on scalar $j_{\sigma}$, assumes that all FACs are crossed perpendicularly $(\alpha=0)$ so that the differences in SwA and SwC equatorial crossing times $\tau_{\text {eq }}$ are used as proxies for $\tau_{0}$ (Forsyth et al 2017, see also Fig. 5F, black). Here, we used the VALOR-determined $\tau_{0}$ for the reference analysis in order to facilitate the comparison.

Next, we cross-check the inclination obtained for the sample event with a constraint $\left(\bar{B}^{*} \cdot \hat{\boldsymbol{n}}=0\right)$ Minimum Variance Analysis (MVA; Sonnerup and Scheible 1998), a technique commonly used to infer the orientation of auroral FACs (e.g., Vogt et al 2020, section 4.4.1). The MVA-inferred normal and polarization directions essentially coincide with the X- and Y-axes of the MFA coordinate frame (Fig. 9) meaning that the inclination is considerably smaller $\left(\alpha_{\mathrm{MVA}}=3.3^{\circ}\right)$ than what is obtained with VALOR $\left(\alpha=19^{\circ}\right)$. Also, the MVA eigenvalue ratio is much higher $\left(\lambda_{\max } / \lambda_{\min }=100\right)$ than the planarity measure we've constructed ( $N$, Fig. 5 ). We don't think that MVA's negligence of the ambient magnetic field variability along the orbits $\left(\measuredangle\left(\boldsymbol{B}, \overline{\boldsymbol{B}}^{*}\right)=6.6^{\circ} \pm 3.8^{\circ}\right)$ is the sole cause for these differences. For now, we attribute them to the spatial resolution power of the respective methods with MVA targeting the orientation of the entire sheet structure while VALOR extracts the local geometry around $t_{0}$.

The purpose of the statistical study is to demonstrate that VALOR can be readily applied to a vast range of auroral oval crossings in a meaningful and largely automated way. We find that the presented results reflect the current knowledge of auroral FAC characteristics in the following sense. First, the MLT asymmetry in center quasi-dipole latitudes (Fig. 5a) is consistent with the ellipsoidal shape of the auroral ovals being squeezed towards and stretched away from the magnetic poles at noon and midnight, respectively (Xiong et al 2014 Xiong and Lühr 2014). Second, the presence of a systematic relation between scale length and MLT, i.e., large-scale FACs $(\geq 300 \mathrm{~km})$ dominate at dawn/dusk while smallscale FACs $(\leq 150 \mathrm{~km})$ dominate at noon/midnight (Fig. 5b, g) is corroborated by McGranaghan et al (2017). Lastly, we find some evidence that the low-latitude FACs are better ordered along the auroral oval (smaller $\gamma$ ) than the high-latitude ones (additional file 1, Fig. 1) although we do not bisect the orbit segments into Region-1 and Region-2 latitudinal parts as do Yang et al (2018).

Regarding the event selection process it is worth to deliberate on the suitability of $j_{12}$ as decisive measure. While its smoothness compared to the single-spacecraft estimates helps the automated determination of the analysis windows, it is not available in the so-called exclusion zones near the geographic poles (lat. $>86^{\circ}$ ) due to the degeneracy of the underlying virtual four-point configuration to a quasi-linear array. 


\section{Conclusions} noon/midnight.

"Loosing" auroral oval crossings due to the exclusion zones could be avoided by estimating current density based on a least-squares approach (e.g., Blăgǎu and Vogt 2019) that is conveniently formulated in the RASADA framework (Vogt et al 2020). The other critical point is the absence of a minimum amplitude requirement for $j_{12}$ which exposes the results to potentially unreliable contributions from signals just above instrument noise. We've tested the influence of FAC density on the results and conclude that low density FACs preferably occur at dawn/dusk with large characteristic scales while high density FACs are prevalent at noon/midnight with smaller scales, respectively (additional file 1, Fig. 2). Excluding the low-intensity FACs would thus lead to an amplification of the already noticeable oversampling at

Moving forward the application of VALOR to SwA and SwC $50 \mathrm{~Hz}$ high-resolution magnetic field observations suggests itself in order to adequately probe the regime of mesoscale auroral arcs (few tens of $\mathrm{km}$ ) or even small-scale (below one $\mathrm{km}$ width) dynamic structures with sufficient East-West elongation $(\sim 200 \mathrm{~km})$. New camera technologies allow to routinely image these aurorae (e.g., Kataoka et al 2021) but their relation to large-scale "background" FACs is not yet fully understood (e.g., Wu et al 2020). With respect to this problem VALOR can be optimized in terms of the specific similarity measure used (e.g., changing the average time scale of MSD in the denominator). Regardless of the application case an important task is to define an easily interpretable quality measure that indicates how well the tuple $\left(t_{0}, \tau_{0}\right)$ can be isolated, e.g., by adopting the concept of topographic prominence. A complementary and independent way to judge VALOR's validity is to properly incorporate concurrent optical data (e.g., from the EMCCD cameras in the MIRACLE network). Of course, VALOR is technically neither limited to the auroral zone nor to Swarm. It can be applied to any multi-spacecraft geospace mission with suitable instrumentation and orbit configuration, e.g., the Magnetospheric Multiscale mission in the plasma sheet boundary layer (Nakamura et al 2018).

We have introduced the methodological framework VALOR for the investigation of field-aligned current (FAC) sheets based on dual spacecraft magnetic field observations. The method was illustrated by means of a sample event (March 1, 2019, 01:58:49-02:03:49 UT), consisting of two consecutive FAC sheet crossings (down and up), regarding which we conclude the following:

- Relatively high levels of vectorial association are found for an optimum spacecraft lag of -11 s and 
385 The authors declare that they have no competing interests. MVA: large-scale) of the respective methods. data base of FAC characteristics that we plan to explore in future studies.

\section{Declarations}

\section{Availability of data and materials} nature of the magnetic input data helps to constrain the center position (Fig. 3).

- The center position falls in between the individual FAC traversals and within the field of view of the Kuujjuaq all-sky camera that concurrently observes a relatively stable auroral arc (southward drifting at $\sim 10 \%$ of spacecraft velocity, Fig. 4). Given the bi-modal nature of the pattern function an interpretation of the center position as the auroral oval center is justified.

- The reference analysis, i.e., linear correlation \& regression according to Forsyth et al (2017), does not indicate large overall similarity at $t_{0}$ but rather small- to meso-scale $(<305 \mathrm{~km})$ fluctuations of matching amplitude (Fig. 7) to which we ascribe VALOR's choice of $t_{0}$. The VALOR determined characteristic length scale $L_{n}=345 \mathrm{~km}$ is corroborated by the reference analysis.

- The sample event presents a typical case of a low-latitude, evening-sector, large-scale auroral current sheet (Fig. 5, 6) with average inclination $\left(\alpha=19^{\circ}\right)$ and convincingly large planarity. We attribute the differences between VALOR and MVA to different inherent resolution powers (VALOR: small-scale,

The fact that VALOR can reproduce known characteristics of auroral FACs (i.e., MLT vs. quasi-dipole latitude occurrence pattern, systematic relation between scale length and magnetic local time) lends credence to the overall capability of the approach. The statistical analysis delivered a comprehensive

The datasets used and/or analysed during the current study are available from the following repositories. Swarm data and derived products are available from https://earth.esa.int/eogateway/missions/swarm/data. The CHAOS-7 geomagnetic field model is hosted at http://www.spacecenter.dk/files/magnetic-models/CHAOS-7. Keograms from the THEMIS All-Sky imagers are accessible via http://themis.ssl.berkeley.edu. 
Funding

LP, JV and NS acknowledge funding by the Deutsche Forschungsgemeinschaft within the priority program 1788 DynamicEarth under grant VO 855/4-1. AB was supported by the ESA project MAGICS, PRODEX contract No. 4000127660.

\section{Authors' contributions}

LP designed the study, derived the results, and lead the writing of the manuscript. JV developed the VALOR approach and participated in the writing of the manuscript. AB contributed to the design of the study and performed a reference MVA analysis. NS implemented the linear correlation/regression analysis. All authors read and approved the final manuscript.

\section{Acknowledgments}

The authors wish to thank the creators of the following open-source Python packages that facilitated the analysis of the data: VirES for Swarm (Smith and pacesm 2020), ChaosMagPy $(\overline{K l o s s} \mid 2020)$, and apexpy (Emmert et al 2010, van der Meeren et al 2018). LP thanks Carsten Baumann for his constructive comments on the manuscript.

Appendix A. The Mean Squared Deviation in terms of Pearson's correlation coefficient and the slope of a linear regression line

We consider two real-valued vectors (time series), $X=\left\{x_{1}, x_{2}, \ldots, x_{N}\right\}$ and $Y=\left\{y_{1}, y_{2}, \ldots, y_{N}\right\}$, and choose the inner product

$$
\langle X \mid Y\rangle=\frac{1}{N} \sum_{n} x_{n} y_{n} .
$$

Then, we get the following expressions for

- a unit vector: $\langle e \mid e\rangle=1$ for $e=\{1,1, \ldots, 1\}$,

- the mean: $\bar{X}=\langle X \mid e\rangle$ (scalar) and $X_{\|}=\langle X \mid e\rangle e$ (vector),

- the variance: $\Delta X^{2}=\left\|X_{c}\right\|^{2}=\left\langle X_{c} \mid X_{c}\right\rangle$ with $X_{c}=X-X_{\|}$,

- Pearson's corr. coeff.: $r(X, Y)=\cos \measuredangle\left(X_{c}, Y_{c}\right)$,

- Mean Squared Deviation: $\operatorname{MSD}(X, Y)=\|X-Y\|^{2}=\langle X-Y \mid X-Y\rangle$. 


$$
\begin{aligned}
\operatorname{MSD}\left(X_{c}, Y_{c}\right) & =\left\|X_{c}-Y_{c}\right\|^{2}=\frac{1}{N} \sum_{n}\left(x_{c, n}-y_{c, n}\right)^{2} \\
& =\left\langle X_{c} \mid X_{c}\right\rangle+\left\langle Y_{c} \mid Y_{c}\right\rangle-2\left\langle X_{c} \mid Y_{c}\right\rangle \\
& =\Delta X^{2}+\Delta Y^{2}-2 r(X, Y) \Delta X \Delta Y .
\end{aligned}
$$

$$
r(X, Y)=\frac{\left\langle X_{c} \mid Y_{c}\right\rangle}{\Delta X \Delta Y}=\frac{\Delta Y}{\Delta X} m \Longleftrightarrow r \Delta X=m \Delta Y,
$$

we can rewrite $\operatorname{MSD}(X, Y)$ as:

$$
\begin{aligned}
\operatorname{MSD}(X, Y) & =\Delta X^{2}\left(1-r^{2}\right)+\Delta Y^{2}(1-m)^{2}+(\bar{X}-\bar{Y})^{2} \\
& =\Delta X^{2}-\Delta X^{2} r^{2}+\Delta Y^{2}-2 m \Delta Y^{2}+m^{2} \Delta Y^{2}+(\bar{X}-\bar{Y})^{2} \\
& =\Delta X^{2}-\Delta X^{2} \vec{r}^{2}+\Delta Y^{2}-2 r(X, Y) \Delta X \Delta Y+r^{2} \Delta X^{2}+(\bar{X}-\bar{Y})^{2}
\end{aligned}
$$

\section{References}

Blăgău A, Vogt J (2019) Multi-Point Field-Aligned Current Estimates with Swarm. Journal of Geophysical Research: Space Physics 124, DOI 10.1029/2018JA026439

Bunescu C, Marghitu O, Constantinescu D, Narita Y, Vogt J, Blăgău A (2015) Multiscale

Field-Aligned Current Analyzer. Journal of Geophysical Research: Space Physics 120:9563-9577, DOI 10.1002/2015JA021670

Bunescu C, Marghitu O, Vogt J, Constantinescu D, Partamies N (2017) Quasiperiodic Field-Aligned Current Dynamics Associated with Auroral Undulations During a Substorm Recovery. Journal of Geophysical Research: Space Physics 122:3087-3109, DOI 10.1002/2016JA023251 
Bunescu C, Vogt J, Marghitu O, Blagau A (2019) Multiscale estimation of the field-aligned current density. Annales Geophysicae 37(3):347-373, DOI 10.5194/angeo-37-347-2019

Emmert JT, Richmond AD, Drob DP (2010) A computationally compact representation of Magnetic-Apex and Quasi-Dipole coordinates with smooth base vectors. Journal of Geophysical Research: Space Physics 115(A8), DOI 10.1029/2010JA015326

Finlay CC, Kloss C, Olsen N, Hammer M, Toeffner-Clausen L, Grayver A, Kuvshinov A (2020) The CHAOS-7 geomagnetic field model and observed changes in the South Atlantic Anomaly. Earth Planets and Space 72, DOI 10.1186/s40623-020-01252-9

Forsyth C, Rae IJ, Mann IR, Pakhotin IP (2017) Identifying intervals of temporally invariant field-aligned currents from Swarm: Assessing the validity of single-spacecraft methods. Journal of Geophysical Research: Space Physics 122(3):3411-3419, DOI 10.1002/2016JA023708

Gjerloev JW, Ohtani S, Iijima T, Anderson B, Slavin J, Le G (2011) Characteristics of the terrestrial field-aligned current system. Annales Geophysicae 29(10):1713-1729,

DOI 10.5194/angeo-29-1713-2011

He M, Vogt J, Lühr H, Sorbalo E, Blagau A, Le G, Lu G (2012) A high-resolution model of field-aligned currents through empirical orthogonal functions analysis (MFACE). Geophysical Research Letters 39(18), DOI 10.1029/2012GL053168

He M, Vogt J, Lühr H, Sorbalo E (2014) Local time resolved dynamics of field-aligned currents and their response to solar wind variability. Journal of Geophysical Research: Space Physics 119(7):5305-5315, DOI 10.1002/2014JA019776

Iijima T, Potemra TA (1976) The amplitude distribution of field-aligned currents at northern high latitudes observed by Triad. Journal of Geophysical Research 81(13):2165-2174, DOI 10.1029/JA081i013p02165

Kataoka R, Chaston C, Knudsen D, Lynch KA, Lysak RL, et al (2021) Small-scale dynamic aurora. Space Sci Rev DOI 10.1007/s11214-021-00796-w

Kloss C (2020) ancklo/ChaosMagPy: ChaosMagPy Version v0.6. Zenodo DOI 10.5281/zenodo.3352398

Knudsen DJ, Donovan EF, Cogger LL, Jackel B, Shaw WD (2001) Width and structure of mesoscale optical auroral arcs. Geophysical Research Letters 28(4):705-708, DOI 10.1029/2000GL011969

Laundal KM, Richmond AD (2017) Magnetic Coordinate Systems. Space Sci Rev 206:27-59, DOI 10.1007/s11214-016-0275-y 
Lotko W, Sonnerup BUO, Lysak RL (1987) Nonsteady boundary layer flow including ionospheric drag and parallel electric fields. Journal of Geophysical Research: Space Physics 92(A8):8635-8648, DOI 10.1029/JA092iA08p08635

Lühr H, Warnecke JF, Rother MKA (1996) An algorithm for estimating field-aligned currents from single spacecraft magnetic field measurements: a diagnostic tool applied to Freja satellite data. IEEE Transactions on Geoscience and Remote Sensing 34(6):1369-1376, DOI 10.1109/36.544560

Lühr H, Park J, Gjerloev JW, Rauberg J, Michaelis I, Merayo JMG, Brauer P (2015) Field-aligned currents' scale analysis performed with the Swarm constellation. Geophysical Research Letters 42:1-8, DOI 10.1002/2014GL062453

Lyons LR (1980) Generation of large-scale regions of auroral currents, electric potentials, and precipitation by the divergence of the convection electric field. Journal of Geophysical Research: Space Physics 85:17-24, DOI 10.1029/JA085iA01p00017

Lysak RL (1990) Electrodynamic Coupling of the Magnetosphere and Ionosphere. Space Science Reviews 52:33-87, DOI 10.1007/BF00704239

Maggs JE, Davis TN (1968) Measurements of the thicknesses of auroral structures. Planetary and Space Science 16(2), DOI 10.1016/0032-0633(68)90069-X

McGranaghan RM, Mannucci AJ, Forsyth C (2017) A Comprehensive Analysis of Multiscale Field-Aligned Currents: Characteristics, Controlling Parameters, and Relationships. Journal of Geophysical Research: Space Physics 122(12):11,931-11,960, DOI 10.1002/2017JA024742 van der Meeren C, Burrell AG, Laundal KM (2018) apexpy: Apexpy version 1.0.3. Zenodo DOI 10.5281/zenodo.1214207

Mende SB, Harris SE, Frey HU (2008) The THEMIS Array of Ground-based Observatories for the Study of Auroral Substorms. Space Sci Rev 141(357), DOI 10.1007/s11214-008-9380-x

Nakamura R, Varsani A, Genestreti K, Contel O, Nakamura T (2018) Multiscale Currents Observed by MMS in the Flow Braking Region. Journal of Geophysical Research: Space Physics 123:1260-1278, DOI $10.1002 / 2017 J A 024686$

Partamies N, Syrjäsuo M, Donovan E, Connors M, Charrois D, Knudsen D, Kryzanowsky Z (2010) Observations of the auroral width spectrum at kilometre-scale size. Annales Geophysicae 28(3):711-718, DOI 10.5194/angeo-28-711-2010

Paschmann G, Haaland S, Treumann R (2002) Auroral plasma physics. Space Science Reviews 103(1), 


\section{DOI 10.1023/A:1023030716698}

Ritter P, Lühr H (2006) Curl-B Technique Applied to Swarm Constellation for Determining

Field-Aligned Currents. Earth, Planets, and Space 58:463-476

Ritter P, Lühr H, Rauberg J (2013) Determining Field-Aligned Currents with the Swarm Constellation Mission. Earth, Planets, and Space 65:1285-1294, DOI 10.5047/eps.2013.09.006

Sandahl I, Sergienko T, Brändström U (2008) Fine structure of optical aurora. Journal of Atmospheric and Solar-Terrestrial Physics 70(18):2275-2292, DOI 10.1016/j.jastp.2008.08.016

Smith A, pacesm (2020) ESA-VirES/VirES-Python-Client: Version v0.7.0. Zenodo DOI 10.5281 /zenodo. 2554162

Sonnerup BU, Scheible M (1998) Minimum and maximum variance analysis. In: Analysis methods for multi-spacecraft data, ISSI Scientific Report SR-001, pp 185-220

Stasiewicz K, Potemra T (1998) Multiscale current structures observed by Freja. Journal of Geophysical Research: Space Physics 103(A3):4315-4326, DOI 10.1029/97JA02396

Vogt J (2002) Alfvén wave coupling in the auroral current circuit. Surveys in Geophysics 23:335-377, DOI 10.1023/A:1015597724324

Vogt J (2014) Analysis of Data from Multi-Satellite Geospace Missions. In: Freeden W, Nashed MZ, Sonar T (eds) Handbook of Geomathematics, Springer Berlin, pp 1-28, DOI 10.1007/978-3-642-27793-1\-69-1

Vogt J, Haerendel G (1998) Reflection and Transmission of Alfvén Waves at the Auroral Acceleration Region. Geophysical Research Letters 25:277-280, DOI 10.1029/97GL53714

Vogt J, Haaland S, Paschmann G (2011) Accuracy of multi-point boundary crossing time analysis. Annales Geophysicae 29:2239-2252, DOI 10.5194/angeo-29-2239-2011

Vogt J, Sorbalo E, He M, Blagau A (2013) Gradient Estimation Using Configurations of Two or Three Spacecraft. Annales Geophysicae 31:1913-1927, DOI 10.5194/angeo-31-1913-2013

Vogt J, Blagau A, Bunescu C, He M (2020) Ionospheric Multi-Spacecraft Analysis Tools, ISSI Scientific Report Series, vol 17, Springer International Publishing, Cham, chap Local Least Squares Analysis of Auroral Currents, pp 55-81. DOI 10.1007/978-3-030-26732-2_4

Vogt J, Blagau A, Pick L (2020) Robust Adaptive Spacecraft Array Derivative Analysis. Earth and Space Science 7(3):e00,953, DOI 10.1029/2019EA000953

Wu J, Knudsen DJ, Gillies DM, \& Burchill JK (2020) Swarm survey of Alfvénic fluctuations and their 
relation to nightside field-aligned current and auroral arc systems. Journal of Geophysical Research: Space Physics DOI 10.1029/2019JA027220

Xiong C, Lühr H (2014) An empirical model of the auroral oval derived from CHAMP field-aligned current signatures \&ndash; Part 2. Annales Geophysicae 32(6):623-631,

DOI 10.5194/angeo-32-623-2014

Xiong C, Lühr H, Wang H, Johnsen MG (2014) Determining the boundaries of the auroral oval from CHAMP field-aligned current signatures \&ndash; Part 1. Annales Geophysicae 32(6):609-622, DOI 10.5194/angeo-32-609-2014

Yang JY, Dunlop MW, Lühr H, Xiong C, Yang YY, Cao JB, Wild JA, Li LY, Ma YD, Liu WL, Fu HS, Lu HY, Waters C, Ritter P (2018) Statistical Correlation Analysis of Field-Aligned Currents Measured by Swarm. Journal of Geophysical Research: Space Physics 123(10):8170-8184, DOI 10.1029/2018JA025205

\section{Figure legends}

Fig. 1: Data for sample event vs. time, magnetic local time (MLT) and quasi-dipole latitude (QDLat). a) Vectorial magnetic field perturbations measured at Swarm Alpha (subscript A, light colors) and Charlie (subscript C, dark colors) pointing towards N (North), E (East) and C (Center). b) Current density according to the Swarm Level-1b single-spacecraft $\left(j_{\mathrm{A}, \mathrm{C}}\right.$, blue $)$ and Level-2 dual-spacecraft $\left(j_{\mathrm{AC}}\right.$, orange) products. c) Probability density (black) and cumulative density (red) functions derived from $j_{\mathrm{AC}}$ with median position $\left(t_{0 i}\right.$, red dashed) and interquartile range (IQR, gray shaded). Black dashed lines mark the final $300 \mathrm{~s}$ long event window boundaries.

Fig. 2: Sketch of observation geometry.

Sketch of a northern hemispheric Field-Aligned Current sheet (FAC, light gray, aligned with ambient magnetic field $\hat{\boldsymbol{q}}$ ) being crossed by Swarm Alpha (SwA) and Charlie (SwC) on orbit segments forming the tangential plane $\left(\hat{\boldsymbol{r}}_{\perp}\right.$, blue) under inclination $\alpha$. a) Special (simple) case where the tangential plane coincides with $\hat{\boldsymbol{q}}_{\perp}$. The association delivers an optimum lag $\tau_{0}$ at center time $t_{0}$ which isolates the polarization direction $\left(\hat{\boldsymbol{p}}_{0}\right.$, red $)$ from the candidates $\left(\hat{\boldsymbol{p}}_{c}\right)$ and determines the sheet normal $\left(\hat{\boldsymbol{n}}_{0}\right.$, red $)$ along which the spatial scale $\left(L_{n}\right)$ is measured. $\underline{\mathbf{b})}$ General case where the tangential plane is rotated away from $\hat{\boldsymbol{q}}_{\perp}$ by the dihedral angle $\beta=\measuredangle(\hat{\boldsymbol{r}}, \hat{\boldsymbol{q}})$. Note that $\hat{\boldsymbol{p}}_{0}$ and $\hat{\boldsymbol{n}}_{0}$ always lie in the $q_{\perp}$-plane 
regardless of the specific viewing geometry.

Fig. 3: Comparison of association measures for the sample event.

A comparison of association measures $\mathcal{C}(t \mid \tau)$ for sample event \#7842 based on single-spacecraft FAC densities $j_{\sigma}\left(\mathcal{C}_{s}\right)$, temporal derivatives of magnetic field perturbations $\dot{\boldsymbol{b}}_{\sigma}\left(\mathcal{C}_{v}\right)$ and a combination of $\dot{\boldsymbol{b}}_{\sigma}$ and the polarization candidates $\hat{\boldsymbol{p}}_{c}\left(\mathcal{C}_{p}\right.$, preferred option). a)-c) Maps of $C(t, \tau)$ normalized to [0,1] with identified optimum lag $\tau_{0}$ indicated by dashed lines. $\underline{\mathbf{d})-\mathbf{f})}$ Association profiles at optimum lag $C\left(t, \tau_{0}\right)$ normalized such that the integral under the curve is one (probability densities, black) and corresponding cumulative densities (red) with median (gray dashed) and interquartile range (IQR, gray shaded).

Fig. 4: VALOR results for the sample event and optical evidence of the associated auroral arc.

a) VALOR orientation results for sample event \#7842 in the X-Y-plane of the mean field-aligned coordinate system. Gray arrows represent the projected ambient magnetic field, contour lines indicate quasi-dipole latitude (vertical) and magnetic local time (horizontal). Colored arrows represent the projected pattern function (Eq. 12) at optimum spacecraft lag along the centered spacecraft locations between event start and end (s, e, black dots). Normalized black arrows show the identified normal and polarization directions at the sheet's center $\left(t_{0}\right.$, red dot). Note that the ambient field is down-scaled with respect to the pattern function. The gray ellipse marks the $9^{\circ}$-wide field of view of the THEMIS All-Sky Imager (ASI) at Kuujjuaq, Canada, at $110 \mathrm{~km}$ altitude. b) Keogram (geographic latitude vs. universal time) constructed from the central North-South slices of the images taken by the Kuujjuaq ASI over a 30-minutes period. Colored lines mark the event boundaries (blue) and center times (preprocessing: orange; VALOR: red) of event \#7842. The shown keogram combines segments from two originals spanning 00:01-00:02 UT and 00:02-00:03 UT.

\section{Fig. 5: Statistical VALOR results.}

Results for 9709 events (04/2014-04/2020). The 2-D histogram in panel a) and the gray bar plots in panels b)-i) indicate VALOR "polarization" results (cf., Fig. 3r, f). The angles in panels d)-f) describe the FAC sheets' orientation in space and are explained in Fig. $2 \mathrm{~b}$ and Fig. 3 a. Here, the colored step functions are stacked and represent two subsets covering the interval $\left[0^{\circ}, 90^{\circ}\right)(\mathrm{cyan})$ and $\left[90^{\circ}, 180^{\circ}\right)$ (magenta). Additional step functions in black, blue and red (not stacked) refer to results obtained either in the preprocessing or from the "scalar" and "vector only" methods, respectively (cf., Fig. 33a, b, 
d, e). Dashed lines mark the corresponding bins for the sample event \#7842.

Fig. 6: Statistical VALOR results in dependence of spatial scale.

Histograms from Fig. 5 (gray) split into three subsets of spatial scales (not stacked).

Fig. 7: Linear correlation and regression analysis applied to the sample event.

Running Pearson's correlation coefficient (panel a) and the slope of the linear regression (panel b) between the filtered single-spacecraft FAC densities from Swarm A and C (lagged by $\tau_{0}=-11 \mathrm{~s}$ ). The window lengths correspond to the smallest periods in the filters which cover the small $(23-152 \mathrm{~km}$; blue), medium (152-305 km; yellow/orange) and large (>305 km; red) spatial scale regimes. Horizontal dashed lines indicate $r=0.9, m=0.9$ and $m=1.1$ levels and red background marks periods at which $r>0.9 \wedge 0.9<m<1.1$ holds for the $40-50$ s filtered signals. Vertical dashed lines indicate the VALOR-determined center time $t_{0}$ and gray background marks the corresponding FAC length scale (IQR). The design of this plot follows that of Forsyth et al (2017).

Fig. 8: VALOR results for the sample event split into two analysis windows.

Same as Fig. 43 but here the event was split at $t_{0}$ and VALOR was applied again to each segment separately. First segment: $t_{01}=02: 00: 36 \mathrm{UT}, \tau_{01}=-4 \mathrm{~s}, L_{n 1}=385 \mathrm{~km}, \alpha_{1}=14^{\circ}, \beta_{1}=169^{\circ}$, $\gamma_{1}=164^{\circ}$. Second segment: $t_{02}=02: 02: 17 \mathrm{UT} \tau_{02}=-10 \mathrm{~s}, L_{n 2}=230 \mathrm{~km}, \alpha_{2}=14^{\circ}, \beta_{2}=166^{\circ}$, $\gamma_{2}=168^{\circ}$

Fig. 9: Magnetic hodogram for sample event.

The blue line tracks the spacecraft centered magnetic field perturbation $\boldsymbol{b}=0.5\left[\boldsymbol{b}_{1}(t)+\boldsymbol{b}_{2, \tau_{0}}(t)\right]$ in the plane perpendicular to the mean ambient magnetic field $\overline{\boldsymbol{B}}^{*}$ from start (s) to end (e). The X- and Y-axes are aligned with the eigenvectors $\boldsymbol{x}_{\mathbf{1}}\left(=\hat{\boldsymbol{p}}_{\mathrm{MVA}}\right)$ and $\boldsymbol{x}_{\mathbf{2}}\left(=\hat{\boldsymbol{n}}_{\mathrm{MVA}}\right)$ to the eigenvalues $\lambda_{1}>\lambda_{2}$ of the data covariance matrix according to a constraint $\left(\overline{\boldsymbol{B}}^{*} \cdot \hat{\boldsymbol{n}}=0\right)$ Minimum Variance Analysis. The VALOR results (black arrows) estimated at $t_{0}$ are shown for reference.

\section{Additional files}

- Additional file 1, "Additional_file_1.pdf", includes two additional figures with legends. 

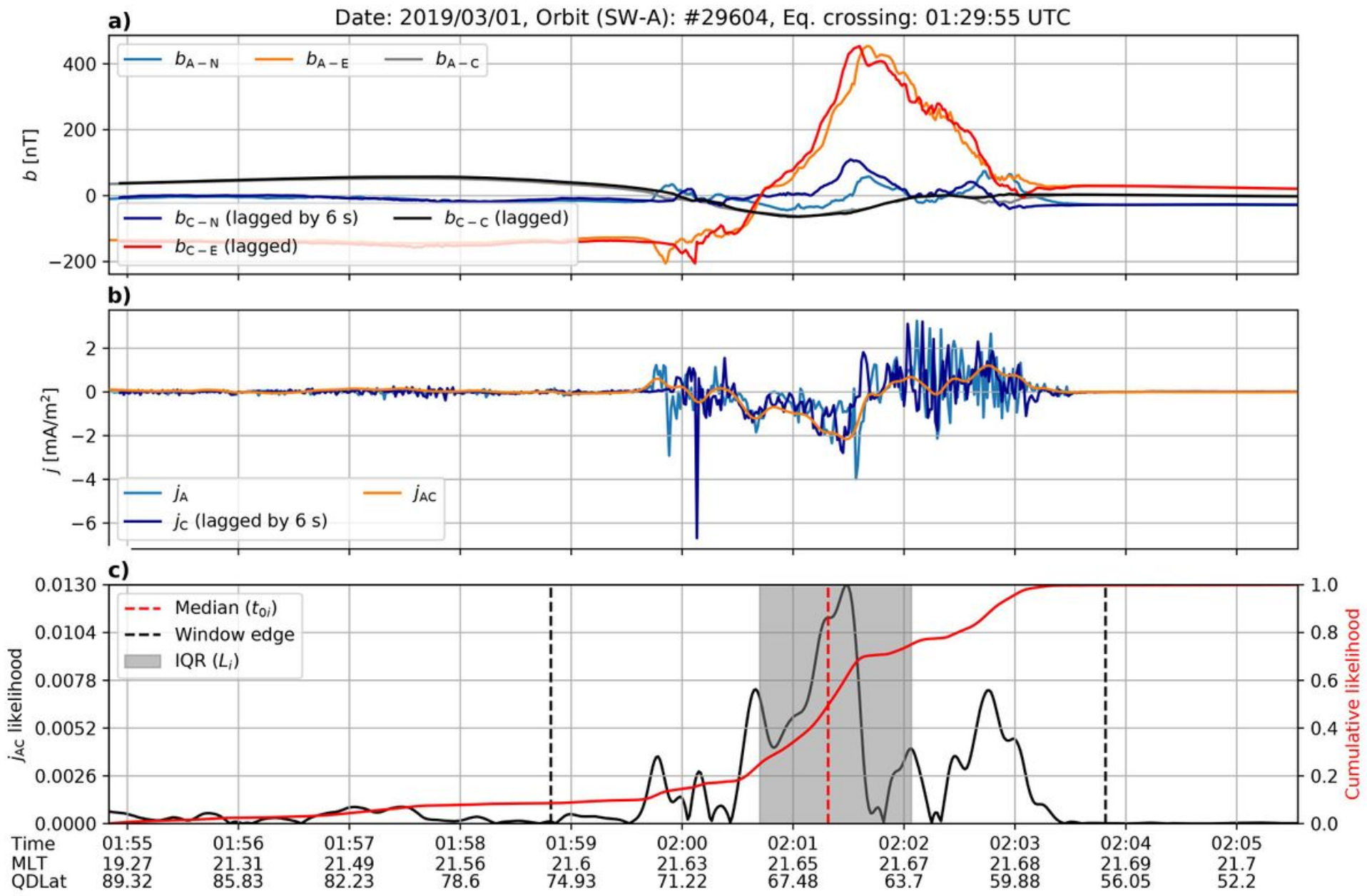

\section{Figure 1}

Please see the Manuscript PDF file for the complete figure caption
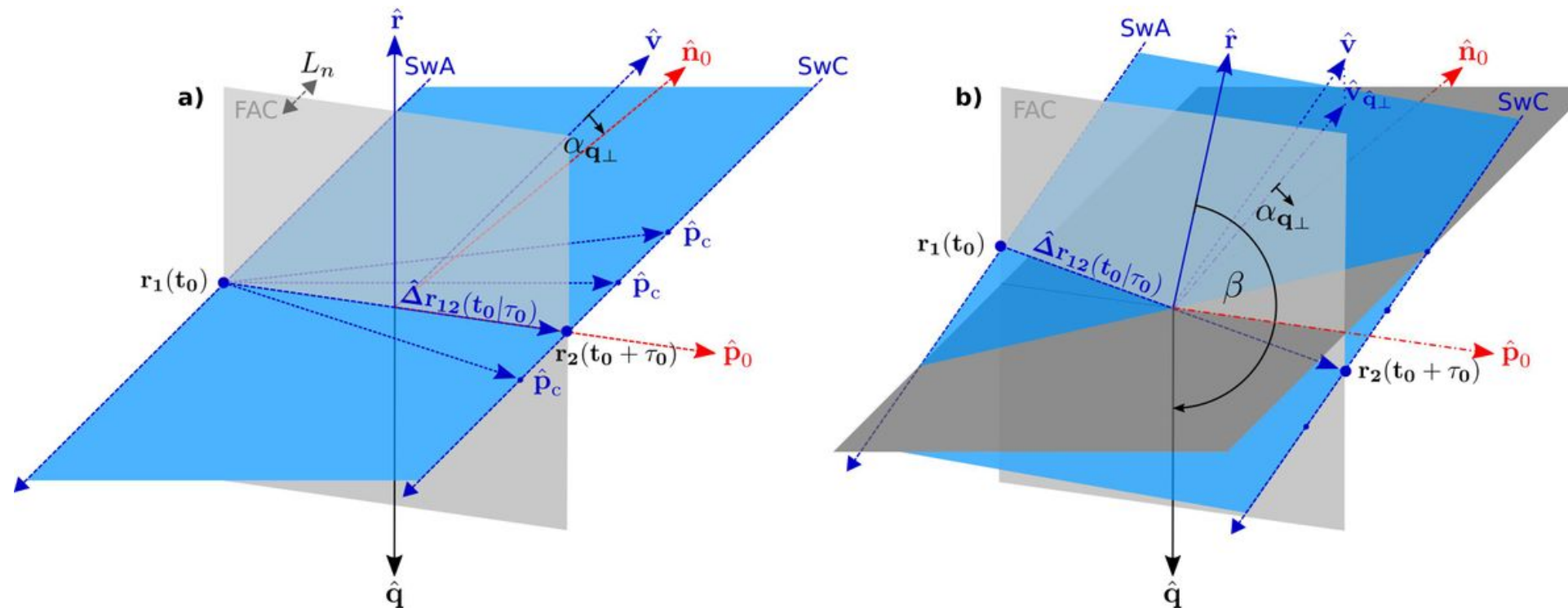
Please see the Manuscript PDF file for the complete figure caption

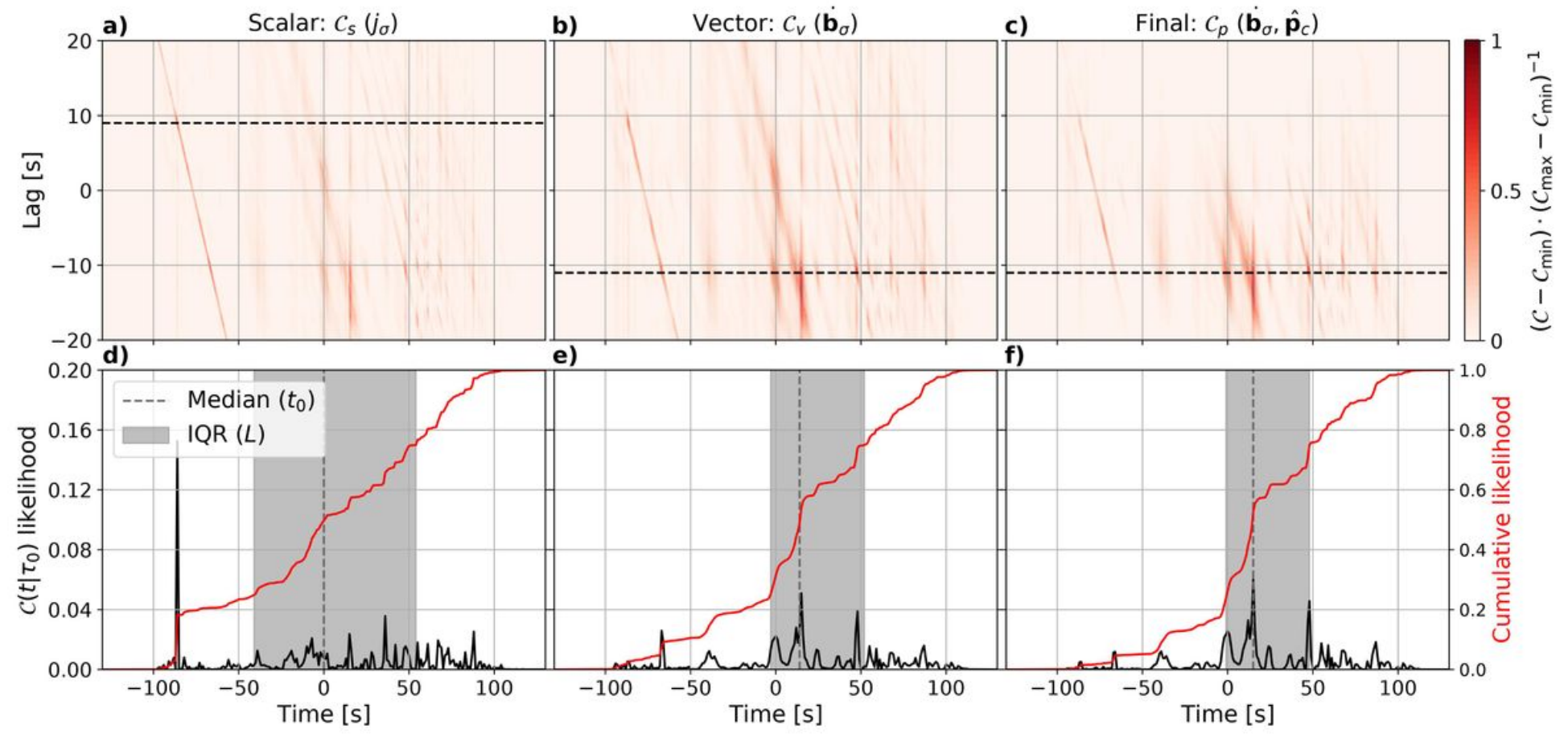

Figure 3

Please see the Manuscript PDF file for the complete figure caption

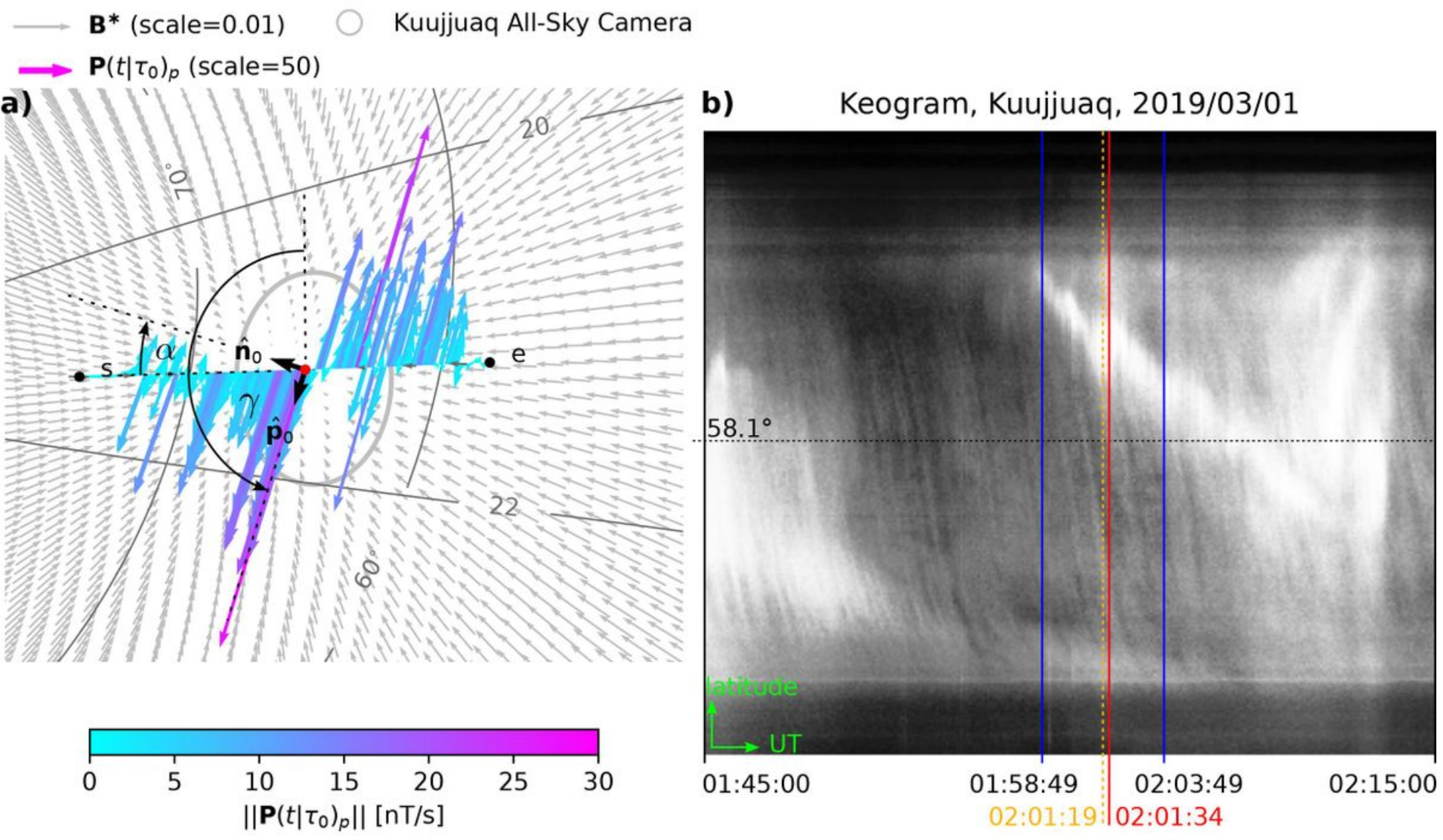

Figure 4 
Please see the Manuscript PDF file for the complete figure caption
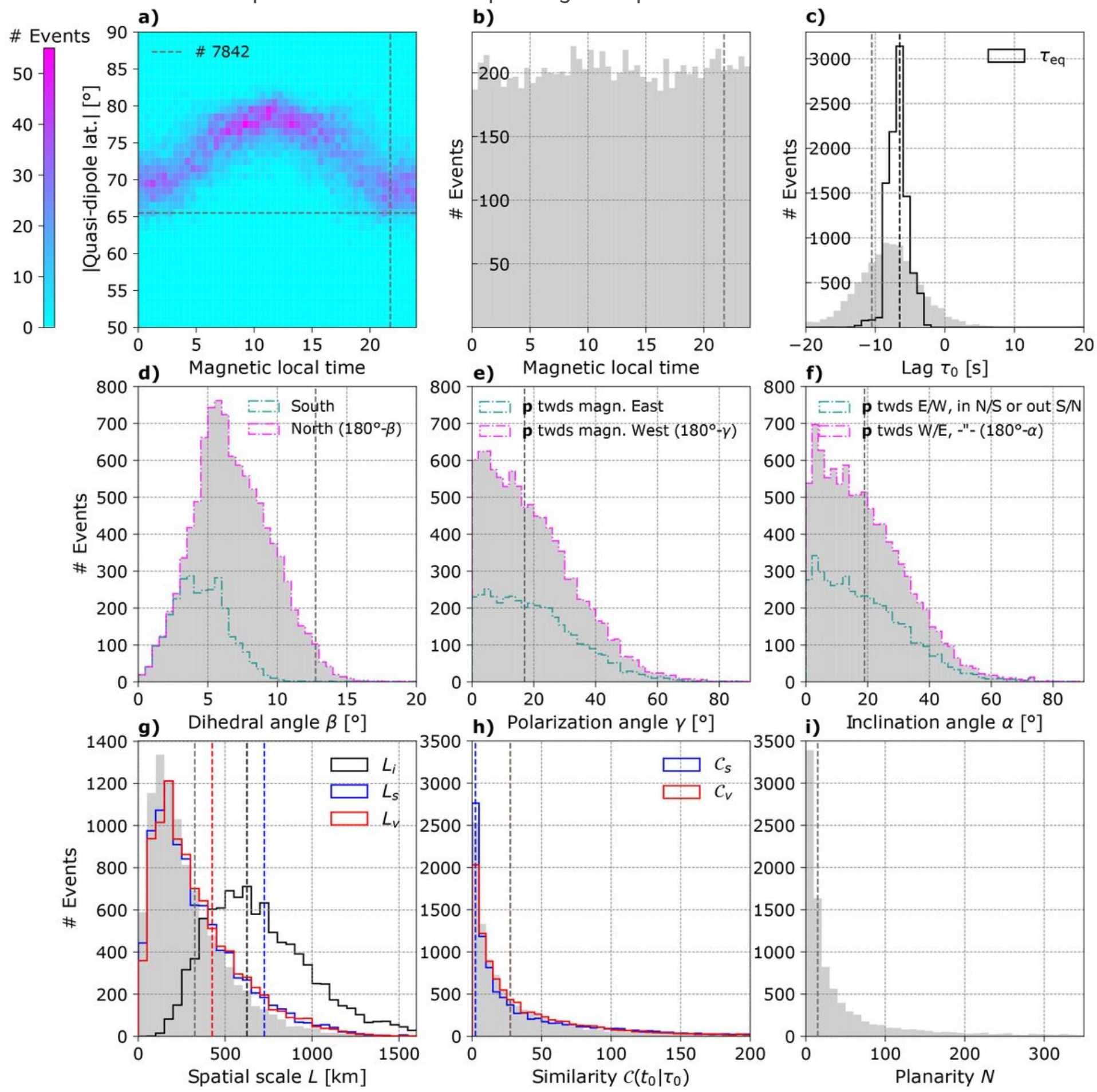

\section{Figure 5}

Please see the Manuscript PDF file for the complete figure caption 


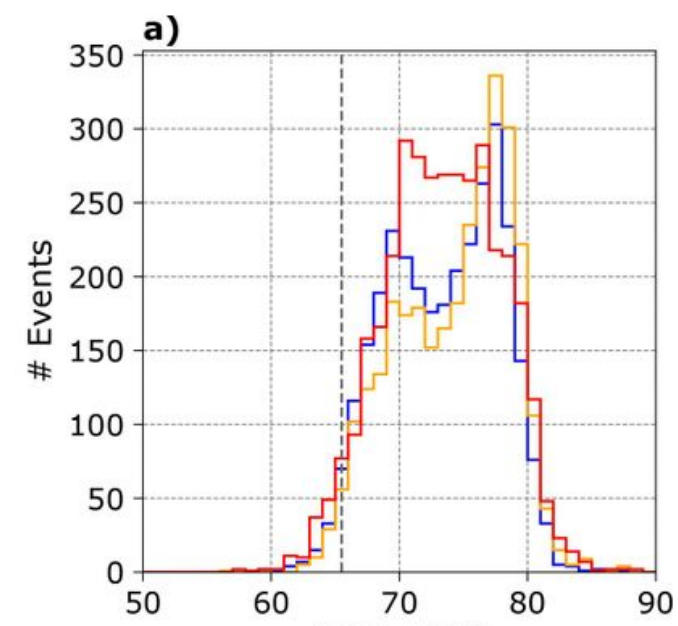

b)
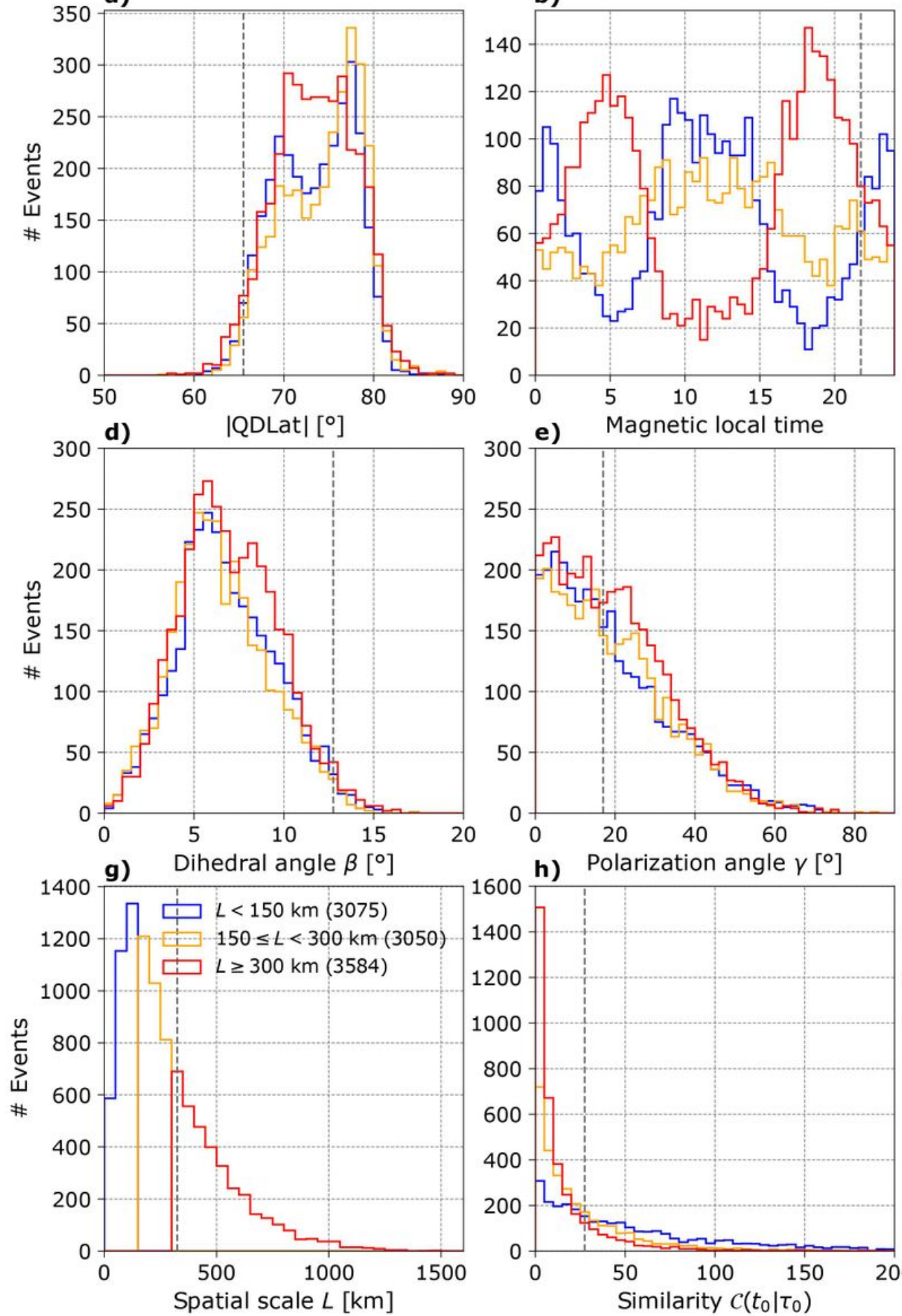

300 e) Magnetic local time
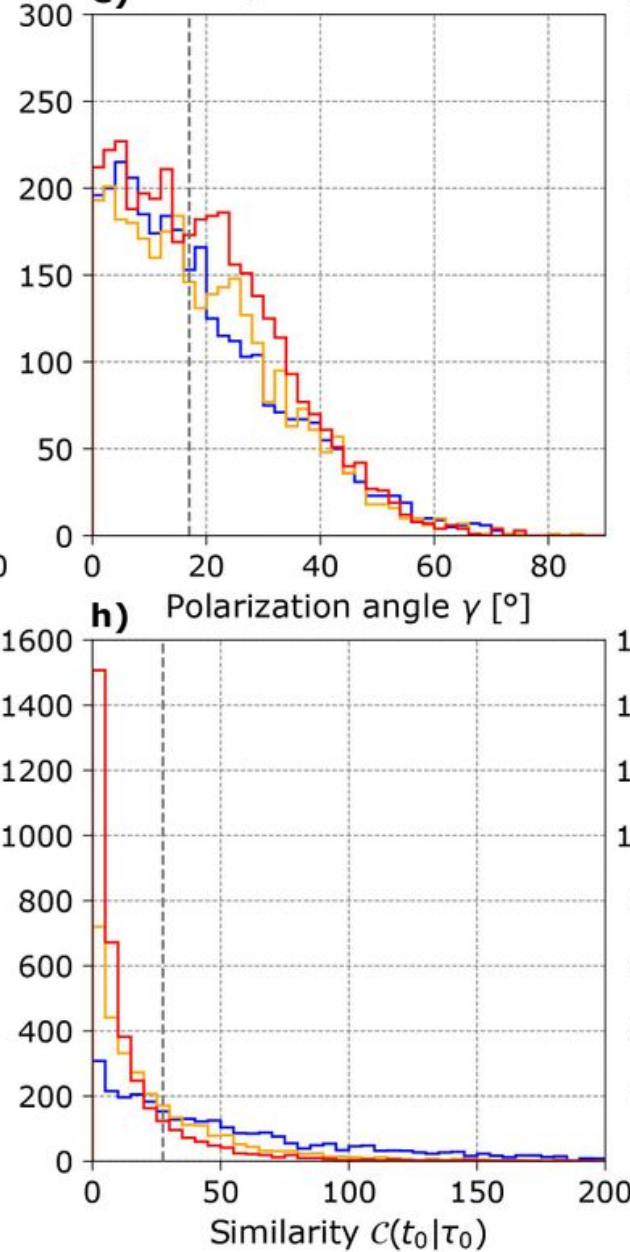

c)
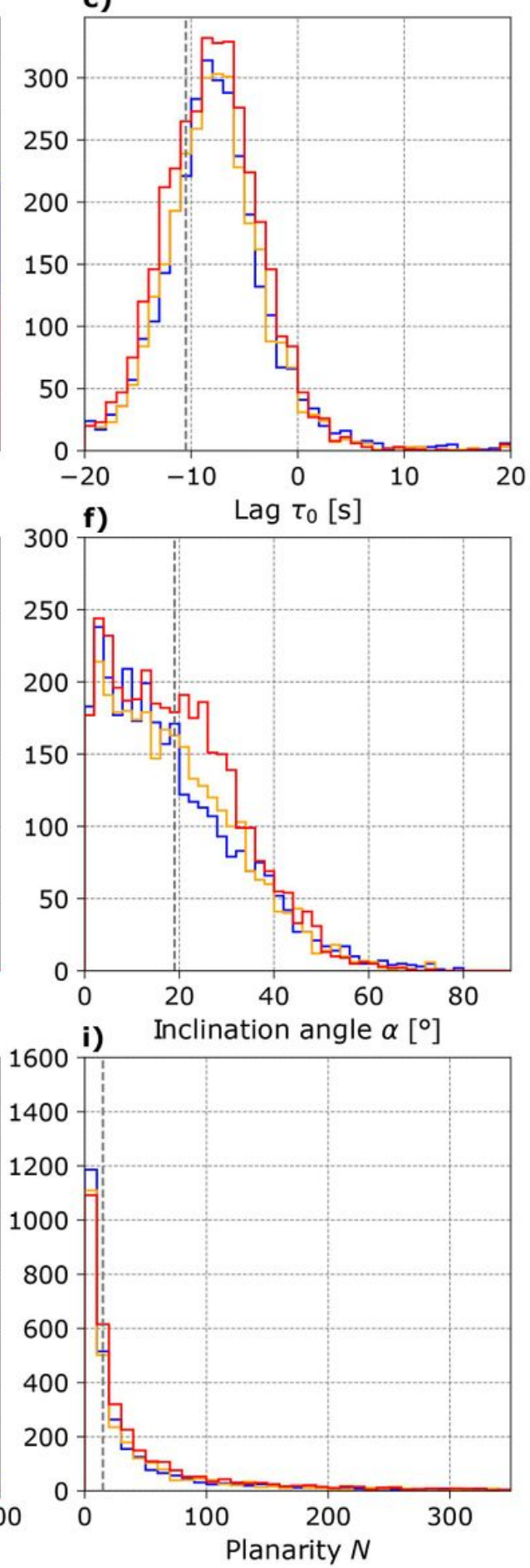

Figure 6

Please see the Manuscript PDF file for the complete figure caption 

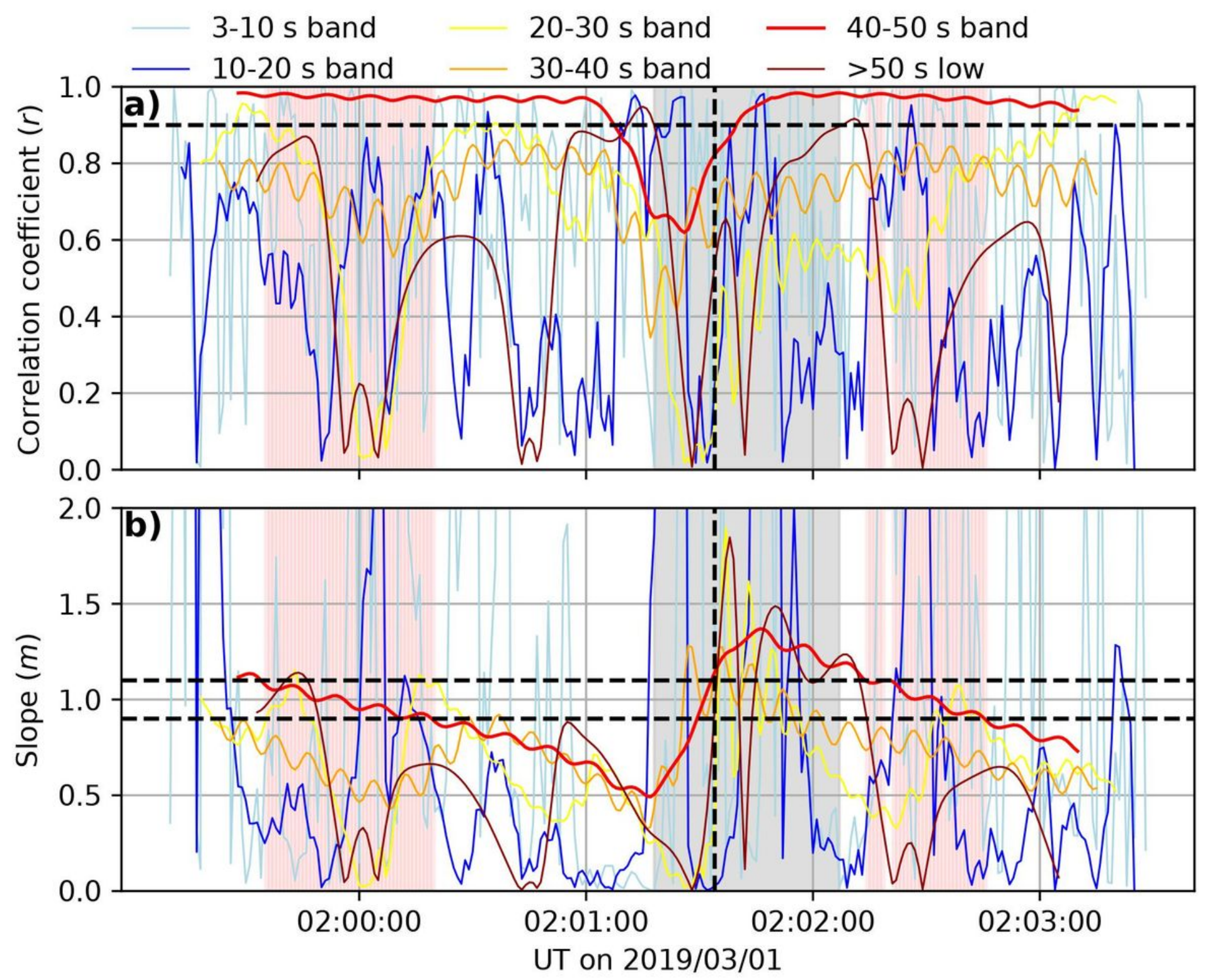

Figure 7

Please see the Manuscript PDF file for the complete figure caption 

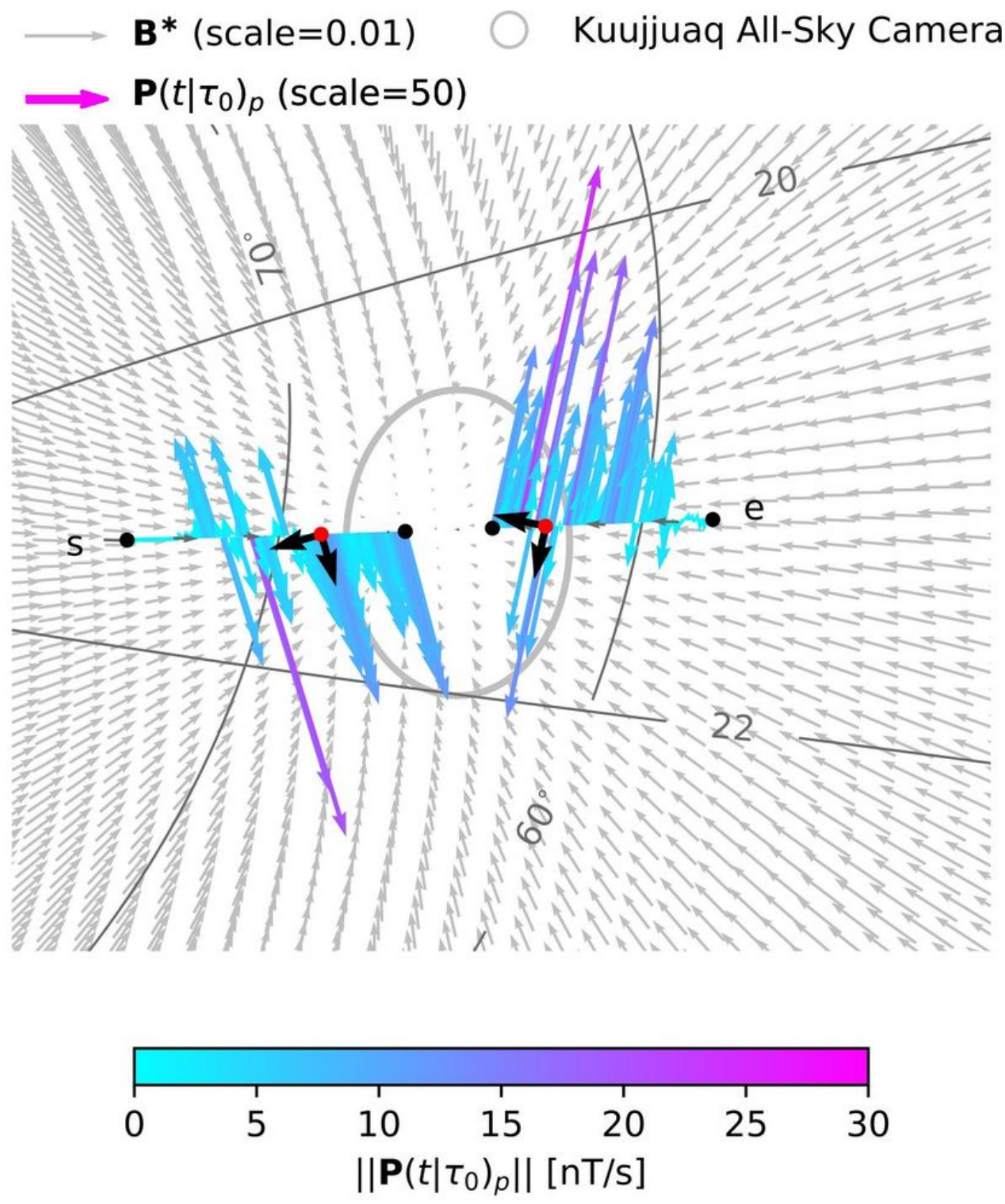

Figure 8

Please see the Manuscript PDF file for the complete figure caption 


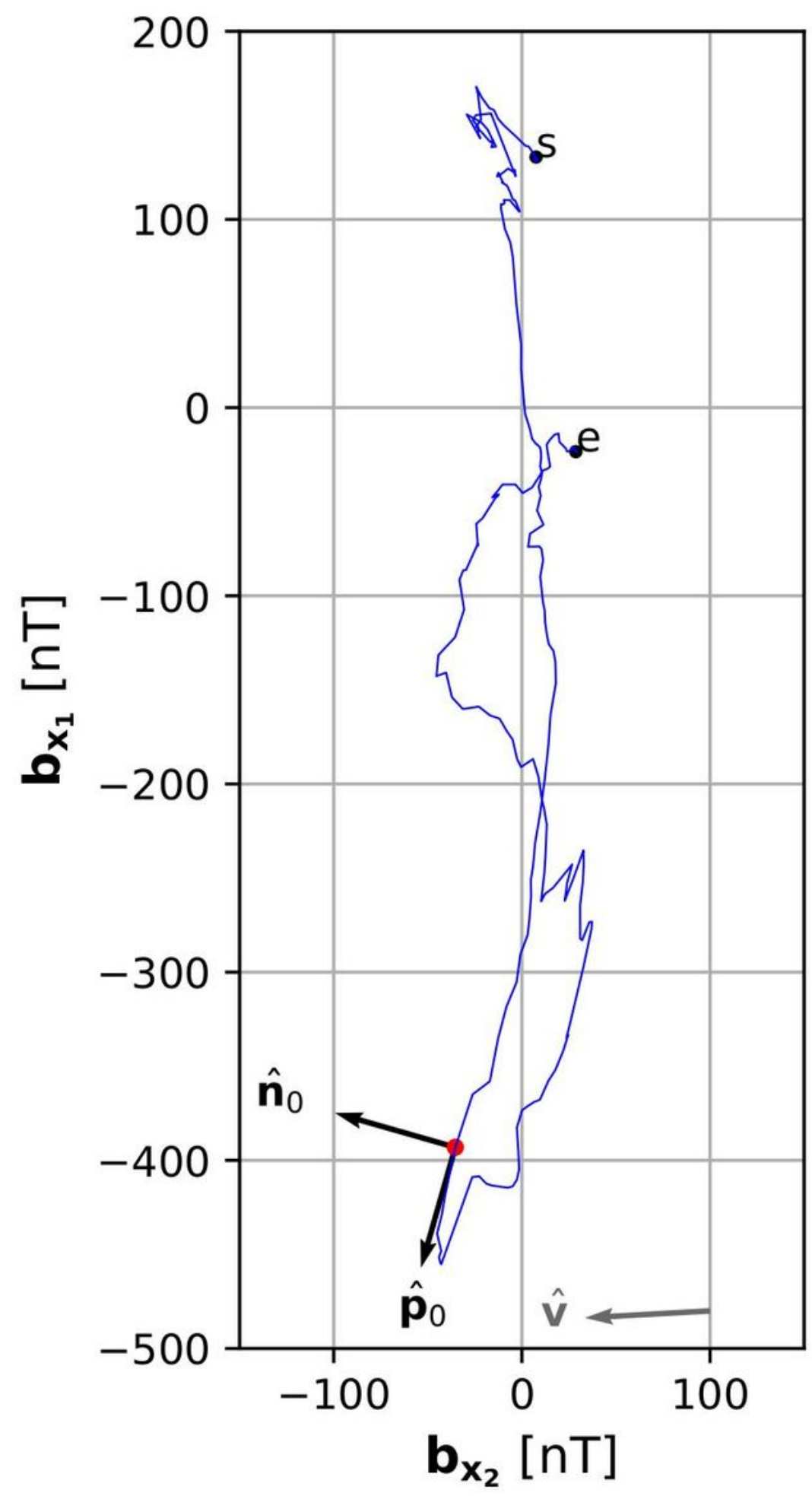

Figure 9

Please see the Manuscript PDF file for the complete figure caption

\section{Supplementary Files}

This is a list of supplementary files associated with this preprint. Click to download. 
- Additionalfile1.pdf

- Graphicalabstract.png 